\title{
A Portrait of the Substrate for Self-Stimulation
}

\author{
C. R. Gallistel \\ University of Pennsylvania
}

\author{
Peter Shizgal \\ Concordia University, Montreal, Canada
}

John S. Yeomans

University of Toronto, Toronto, Ontario, Canada

\begin{abstract}
Quantitative properties of the neural system mediating the rewarding and priming effects of medial forebrain bundle (MFB) stimulation in the rat have been determined by experiments that trade one parameter of the electrical stimulus against another. The first-order neurons in this substrate are for the most part long, thin, myelinated axons, coursing in the MFB and ventral tegmentum, with absolute refractory periods in the range $.5-1.2 \mathrm{msec}$ and conduction velocities of $2-8 \mathrm{~m} / \mathrm{sec}$. Local potentials in these axons decay with a time constant of about $.1 \mathrm{msec}$. A supernormal period follows the recovery from refractoriness. These axons integrate current over exceptionally long intervals, accommodate slowly, and fire on the break of prolonged anodal pulses. These properties rule out the hypothesis that catecholamine pathways constitute the first-order axons. The second-order (postsynaptic) part of the substrate shows surprisingly simple spatial and temporal integrating characteristics. We examine the logic that permits conclusions of this sort to be derived from behavioral data and the role of these derivations in establishing neurobehavioral linkage hypotheses.
\end{abstract}

In this article we describe properties of the neural tissue whose excitation eventuates in the reinforcing and motivating effects of electrical stimulation of the medial forebrain bundle (MFB) in the rat. The goal of the research is to identify these systems by anatomical and electrophysiological methods, thus providing physiological psychology with a model for studying the neurophysiological bases of learning and motivation in a higher vertebrate.

The properties of the substrate for selfstimulation described in this article have been inferred from behavioral trade-off experiments-experiments that determine the

The authors are listed in alphabetical order. They gratefully acknowledge the following grant support: National Science Foundation Grant BMS-16339 to C. Gallistel; National Institute of Health Grant NS-14935 to C. Gallistel, P. Hand, \& M. Reivich; National Institute of Health Training Grant MHI5092 (A. Epstein \& C. Gallistel, codirectors); Natural Science and Engineering Research Council (NSERC) of Canada Grant A0308 and Quebec Formation des Chercheurs et les Actions Concertées (FCAC) Grant EQ-09 to P. Shizgal; and NSERC of Canada Grant A7077 to J. Yeomans.

Requests for reprints should be sent to C. R. Gallistel, Department of Psychology, University of Pennsylvania, 3813-15 Walnut Street, Philadelphia, Pennsylvania 19104. value of one parameter of stimulation (e.g., current intensity) required to produce a criterion level of performance at each setting of another parameter (e.g., pulse duration). We review experiments of this kind in selfstimulation while examining two theoretical questions: (a) Why do behavioral trade-off functions have the power to reveal quantitative properties of the substrate for the behavior? and (b) Why must trade-off experiments play a pivotal role in relating anatomical and electrophysiological data to the behavioral phenomenon?

Using microelectrodes, physiological psychologists have long been able to examine the response of single neurons to a behaviorally significant stimulus, such as rewarding brain stimulation (Rolls, 1975). The advent of 2-deoxyglucose autoradiography makes it possible to visualize a whole range of neural systems activated by such a stimulus (Figure 1). The existence of powerful techniques for directly revealing nervous activity confronts us with a conceptual problem that physiological psychology shares with other reductionist disciplines: How may one establish that a system defined by observations at one level of analysis explains phe- 
nomena at another level? In the present instance, how may we establish that activity in a neural system defined by electrophysiological and anatomical observations is the basis for the self-stimulation phenomenon? Many of the neural systems whose activation is revealed Figure 1 probably have nothing to do with the rewarding effect of the stimulation. How may one support the claim that a particular one of them carries the neural signals mediating the rewarding or motivating effects of the stimulation? This is the theoretical problem to which we speak.

Our answer in brief is that neurophysiological and neuroanatomical techniques are, by themselves, incapable of solving the problem. One must establish by behavioral experiments - and most often by trade-off experiments-what kinds of neurophysiological, neuroanatomical, and neuropharmacological attributes are to be looked for. Only then can electrophysiological and neuroanatomical methods be fruitfully employed to identify the particular system or systems whose excitation leads to self-stimulation.

\section{The Portrait}

Our portrait of the substrate for MFB self-stimulation derives from five basic tradeoff experiments: (a) the required number of pulse pairs as a function of within-pair interval when a train of paired pulses is delivered to a single electrode; (b) the required number of pulse pairs as a function of within-pair interval when the first and second pulses of each pair are delivered via different electrodes; (c) the required current as a function of pulse duration; (d) the required current as a function of the number of pulses in a train of fixed duration; and (e) the required strength of stimulation as a function of train duration. The data from these five experiments may be used to support or reject hypotheses about which of the neural systems in Figure 1 is the substrate for selfstimulation. A neural system hypothesized to underlie MFB self-stimulation must have properties that yield the sorts of trade-off data we here review.

We explain the data from these experiments by postulating that the reinforcing and motivating effects of MFB stimulation are primarily due to the activation of a neural system with the following properties:

1. The self-stimulation-relevant tissue directly excited by the electrode current is a bundle of axons that course through the MFB and the midbrain tegmentum.

2. These axons are myelinated.

3. Most of them have absolute refractory periods in the range $.5-1.2 \mathrm{msec}$.

4. Following the refractory period, there is a period of supernormal excitability, which is most evident in the period $2.0-5.0 \mathrm{msec}$ after an impulse.

5. The first-stage axons conduct impulses at velocities of $2-8 \mathrm{~m} / \mathrm{sec}$.

6. Their diameter is probably $.3-1.5 \mu$.

7. Local nonconducted potentials in these axons decay in an approximately exponential manner with a time constant of about .1 msec.

8. The first-stage axons integrate current over exceptionally long intervals. When a cathodal pulse is applied, the number of firings it produces in the axon bundle continues to increase for up to $15 \mathrm{msec}$.

9. These axons accommodate slowly to an anodal (hyperpolarizing) pulse. They fire on the offset of an anodal pulse longer than 5 msec.

10. The first-stage axons cannot be any of the known catecholamine projections, because the axons comprising these projections have the wrong properties: They are unmyelinated, with refractory periods greater than $1.5 \mathrm{msec}$ and conduction velocities less than $1.0 \mathrm{~m} / \mathrm{sec}$.

11. The number of first-stage axons fired by very short pulses $(.1 \mathrm{msec})$ increases as a linear function of current intensity.

12. The barrage of stimulation-produced action potentials in the first stage passes transsynaptically into a neural network that performs a surprisingly simple temporal and spatial integration: For any given barrage duration, the integrator's output depends solely on the number of action potentials in the barrage, not on their distribution in space and time (provided that the average number of spikes per second is constant during the train).

13. This integrator sums with decreasing 


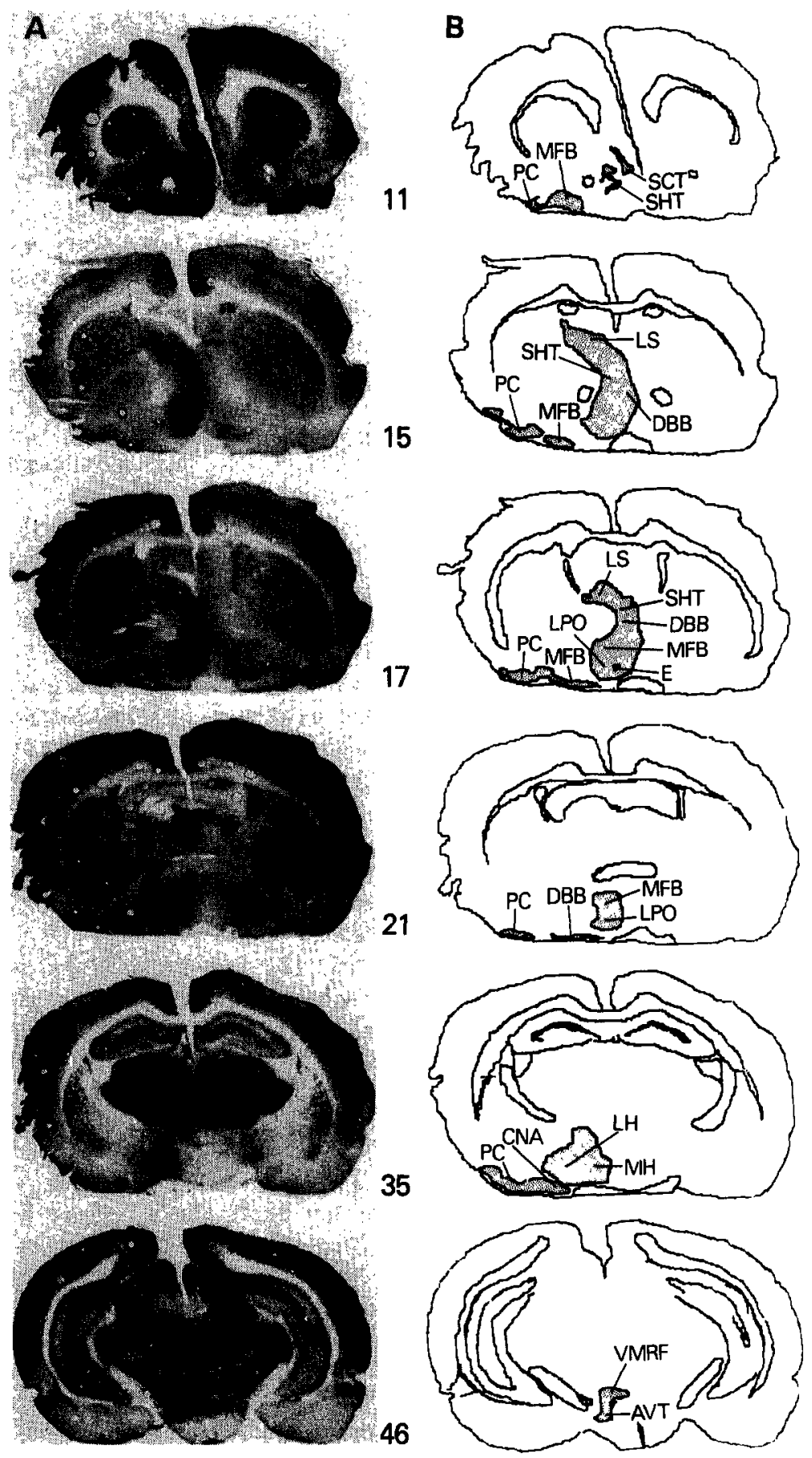

Figure 1. Panel A: Neural systems excited by a self-stimulation electrode on the left side of the rat brain in the medial aspect of the lateral preoptic area are revealed by $\left[{ }^{14} \mathrm{C}\right]-2$-deoxyglucose autoradiography (for technique, see Sokoloff et al., 1977). (Rat was self-stimulating during uptake of the radioactive tracer.) Panel B: Computer assisted outline drawings of the autoradiographs, showing the regions of unilaterally elevated activity (shaded and labeled). (The outlines of the regions of elevated activity were generated by setting the upper limit of a color window [Goochee, Rasband, \& Sokoloff, 1980] so as to just color in the corresponding region on the control [unstimulated] side of the brain, 
efficiency over an interval of up to about $2 \mathrm{sec}$.

14. The number of action potentials required to produce a given level of reward is a linearly increasing function of train duration, which means that the required strength of the barrage (the number of action potentials per second) decreases hyperbolically.

\section{The Minimal Model}

In designing and interpreting the experiments, we worked with the following model of the self-stimulation phenomenon. We term this the minimal model because it seems to us that its assumptions must appear in one form or another in any more fully developed theory of the phenomenon. It is a highly abstracted, bare-bones model that does not address itself to many perplexing and controversial questions. Most of the assumptions we state here are justified by data to be presented. We state them now to make the rationale for the experiments more transparent.

\section{The Reward Pathway}

The cable. We have assumed that the initial neurophysiological stage in the production of a reinforcing effect involves the excitation of action potentials in a bundle of reward-relevant axons surrounding the electrode tip. The reward-relevant axons that can be directly excited by the current comprise the cable. The axons composing the cable may or may not be the same from one electrode locus to the next.

The integrator. It is assumed that action potentials in the cable are not the proximal cause of the reinforcing effect. Rather, these action potentials give rise to a neurophysi- ological signal that must propagate across an unknown number of synapses before it produces the reinforcing effect. The network of neurons and synapses through which the reward signal must propagate is assumed to summate the temporally and spatially distributed effects of the action potentials in the cable. This second stage may have many successive relays of neurons in it, elaborate cross connections, feedback loops, and so forth. No assumptions are made about the circuitry in this second stage, except that it performs temporal and spatial summation. This network is termed the integrator.

It is conceivable that there is no second stage neural network. The cable may terminate at the site where the reinforcing effect is generated. In that event, the term integrator refers to the processes that perform a spatial and temporal summation of the effects of the action potentials in the cable.

The conversion process. In order to influence subsequent behavior, the magnitude of the rewarding signal generated by the stimulation must be recorded. The transient rewarding signal must therefore be converted into an engram, an enduring record of the magnitude of the signal (see Gallistel, Stellar, \& Bubis, 1974, for experimental justification). The site of the conversion process is the site where the reinforcing effect is generated. The output of the integrator, which is by definition the signal seen by the conversion process, is the proximal cause of the reinforcing effect.

The neurophysiological and neuroanatomical identity of the reward pathway is an interesting question in large part because the pathway must lead to an engram-making process. If one knew the pathway and where it led, one might be in a position to study the neurophysiological basis of engram formation in a higher vertebrate.

then running the outlining cursor along the color boundary where the activity rose above the upper level of the window. The numbers in the center give the approximate plate number in the Konig and Klippel [1967] atlas. Abbreviations: AVT = anterior ventral tegmentum; CNA $=$ cortical nucleus of the amygdala; $\mathrm{DBB}=$ diagonal band of Broca; $\mathrm{E}=$ electrode tip; $\mathrm{LH}=$ lateral hypothalamus; $\mathrm{LPO}=$ lateral preoptic area; $\mathrm{LS}=$ lateral septum; $\mathrm{MFB}=$ medial forebrain bundle; $\mathrm{MH}=$ medial hypothalamus; $\mathrm{PC}$ = pyriform cortex; SCT = septocortical tract; $S H T=$ striohypothalamic tract; VMRF = ventromedial reticular formation. [Autoradiographs by J. Yeomans, outlining by C. Gallistel on the image processing facility at Drexel University under the direction of $M$. Negin.]) 


\section{The Priming Pathway}

Rewarding stimulation not only produces an enduring record, it also produces a transient potentiation of behavior rewarded by brain stimulation. Rewarding stimulation delivered just before the animal is allowed to perform the behavior that produces stimulation transiently increases the vigor of that behavior and the likelihood that it will be performed in preference to other competing behaviors (see Gallistel, 1973, for review). Unlike the reinforcing effect, this priming effect does not depend on the stimulation's being contingent on the animal's behavior.

In our minimal model it is assumed that the first stage in the production of the priming effect also involves the direct electrical excitation of action potentials in a bundle of priming-relevant axons coursing near the electrode. The bundle of priming-relevant axons may or may not be one and the same as the bundle of reward-relevant axons. The minimal model is noncommittal on this point, although the data we review show that the reward cable and the priming cables are very similar, if not identical.

The priming pathway must diverge at some point from the reward pathway, since the priming effect is not mediated by engram formation (Gallistel et al., 1974).

The priming effect has all the properties of a strong motivational process. That is, it selectively energizes and directs behavior (see Gallistel, 1975, 1980, for elaboration). Identifying the substrate for the priming process should put us in a position to study the neurophysiological basis for a powerful motivational process in a higher vertebrate.

\section{Summary}

Figure 2 schematizes our minimal model of the self-stimulation substrate. For diagrammatic simplicity, the priming cable and priming integrator are not shown. A schematic rendering of them would be identical to the rendering of the reward cable (first stage neurons in Figure 2) and the reward integrator (second stage in figure 2), except that the output of the priming integrator bypasses the memory-formation process. Whatever priming signal remains as a decaying residue of recent stimulation combines with the engram from previous behavior-contingent stimulations to determine performance on a given trial.

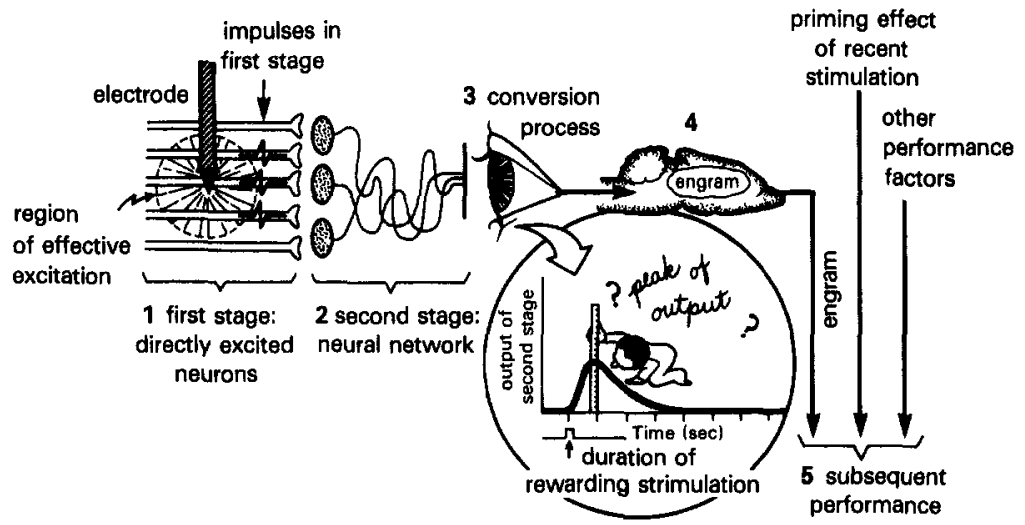

Figure 2. A minimal model of the self-stimulation phenomenon. (The stages by which the rewarding effect is realized in performance are numbered as follows: 1. Stimulating pulses produce successive volleys of impulses in reward-relevant axons. 2. A postsynaptic network of unknown complexity carries out temporal and spatial summation. 3. The transient output of this network is seen by a conversion process, represented by the homunculus, which translates some aspect of this signal [e.g., its peak] into an engram - a record of the reward. 4. This record, together with the priming effect of recent stimulation and innumerable other factors, determines the speed [vigor, rate, etc.] of subsequent performance. For diagrammatic simplicity, the priming pathway is not shown. It would be represented by a directly stimulated first stage and an integrator, but no engram-forming stage. [Adapted from Gallistel, 1978, Figure 9.]) 
Many other variables-health, natural motivational state, time of day, and so oninfluence the vigor of self-stimulation. These other variables, termed performance factors in Figure 2, must be held constant. Meaningful trade-off data are only obtained when the state of the animal does not vary systematically from the determination of one point to the next.

In reviewing the five kinds of experiments, we concentrate first on the reward pathway. We then turn to the priming pathway and the question of whether its quantitative characteristics are in any way distinct.

\section{Trade-Off Experiments}

\section{Paired-Pulse Experiments}

These experiments assess the effectiveness of stimulation with paired pulses as a function of the interval between the first and second pulses (the $\mathrm{C}-\mathrm{T}$ interval). The first pulse in the pair of pulses is called the conditioning or $C$ pulse. The second pulse is called the test or $T$ pulse because it tests the excitability of the substrate in the wake of the neural activity initiated by the first pulse. In other words, paired-pulse experiments measure the poststimulation excitability - the sequence of changes in excitability that a neuron goes through once it has been stimulated. A phase of particular relevance in this cycle is the period of absolute refractoriness following the initiation of a spike. During this absolute refractory period a second spike cannot be initiated.

\section{One-Electrode Experiments}

In the experiments to be reviewed first, rats pressed a bar to obtain a 250 -msec train of paired pulses, and the effectiveness of the stimulation at various $C-T$ intervals was measured. To measure the effectiveness, the number of pairs required to produce a criterial rate of pressing was determined. Since train duration was fixed, the frequency of the pulse pairs covaried with their number. An example of such an experiment is shown in Figure 3, Panel A. There is a narrow range within which the rate of pressing is a steep function of the number of pairs in the train.
Below that range, bar pressing rates are very low; above that range, they are near maximum. To determine the trade-off between the $\mathrm{C}-\mathrm{T}$ interval and the number of pulse pairs, a behavioral criterion half way between maximal and minimal performance is selected-in this case, 40 presses per minute. The trade-off function is the number of pulse pairs required to produce this level of performance at each value of the $\mathrm{C}-\mathrm{T}$ interval.

The less effective the stimulation at a given $\mathrm{C}-\mathrm{T}$ interval, the greater the required number of pulse pairs. The effectiveness of the paired pulses was measured by comparing the required number of pulse pairs, $N(\mathrm{C}$ $\mathrm{T})$, to the number of $\mathrm{C}$ pulses required in the absence of any $T$ pulses, $N($ No- $T)$. When $\mathrm{T}$ pulses are omitted altogether, their effectiveness is obviously zero. If $T$ pulses are present but ineffective, the required number of pulse pairs, $N(\mathrm{C}-\mathrm{T})$, will be the same as the required number of single, unpaired pulses, $N(\mathrm{No}-\mathrm{T})$. If, on the other hand, the $\mathrm{T}$ pulses are fully as effective as the $\mathrm{C}$ pulses, that required number of pulse pairs will be half the required number of single pulses. In other words, as the effectiveness of the $T$ pulse goes from zero (might as well not be there) to unity (just as effective as the C pulse), the ratio $N($ No- $\mathrm{T}) / N(\mathrm{C}-\mathrm{T})$ goes from one to two. These considerations lead to Yeomans's statistic for paired-pulse effectiveness:

$$
[N(\mathrm{No}-\mathrm{T}) / N(\mathrm{C}-\mathrm{T})]-1 .
$$

Similar reasoning leads to a comparable formula for paired-pulse effectiveness in experiments that trade current, $I$, against $\mathrm{C}-$ $\mathrm{T}$ interval:

$$
[I(\mathrm{No}-\mathrm{T}) / I(\mathrm{C}-\mathrm{T})]-1 .
$$

We will argue in a moment that the procedure that varies current instead of number of pulse pairs should only be used in special circumstances.

Figure 3, Panel B shows the biphasic variation in paired-pulse effectiveness when $C$ and $T$ pulses are presented on one electrode and the number of pairs is traded off against the C-T interval (Yeomans \& Davis, 1975). As $\mathrm{C}-\mathrm{T}$ interval increases from zero, effec- 


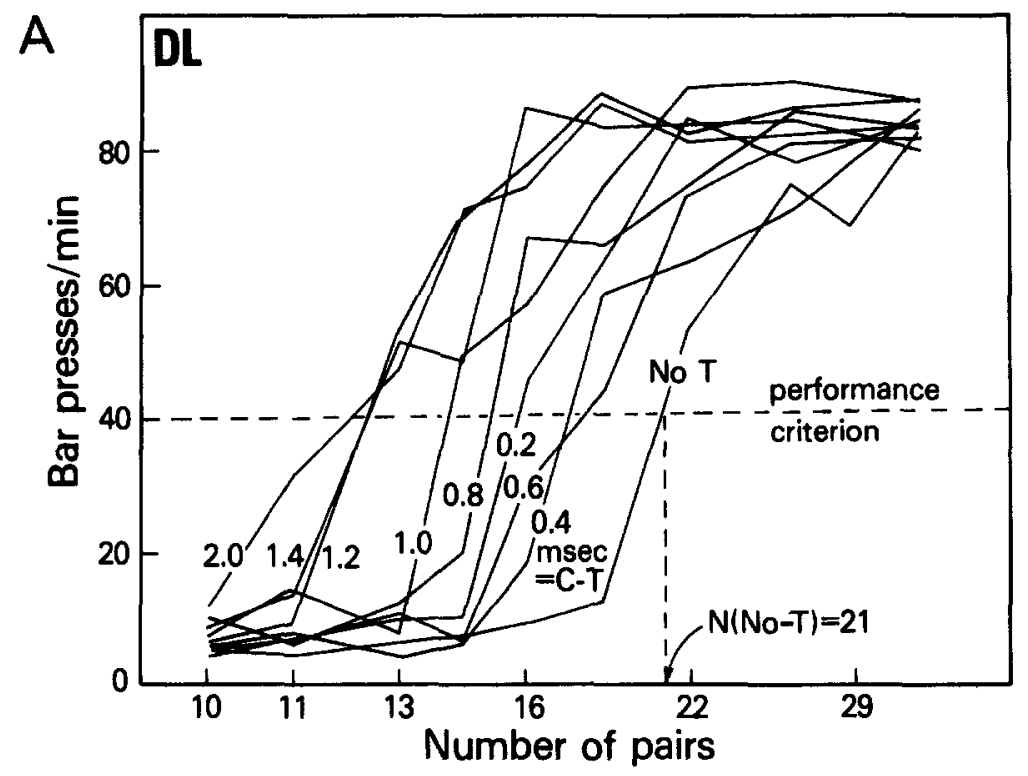

B
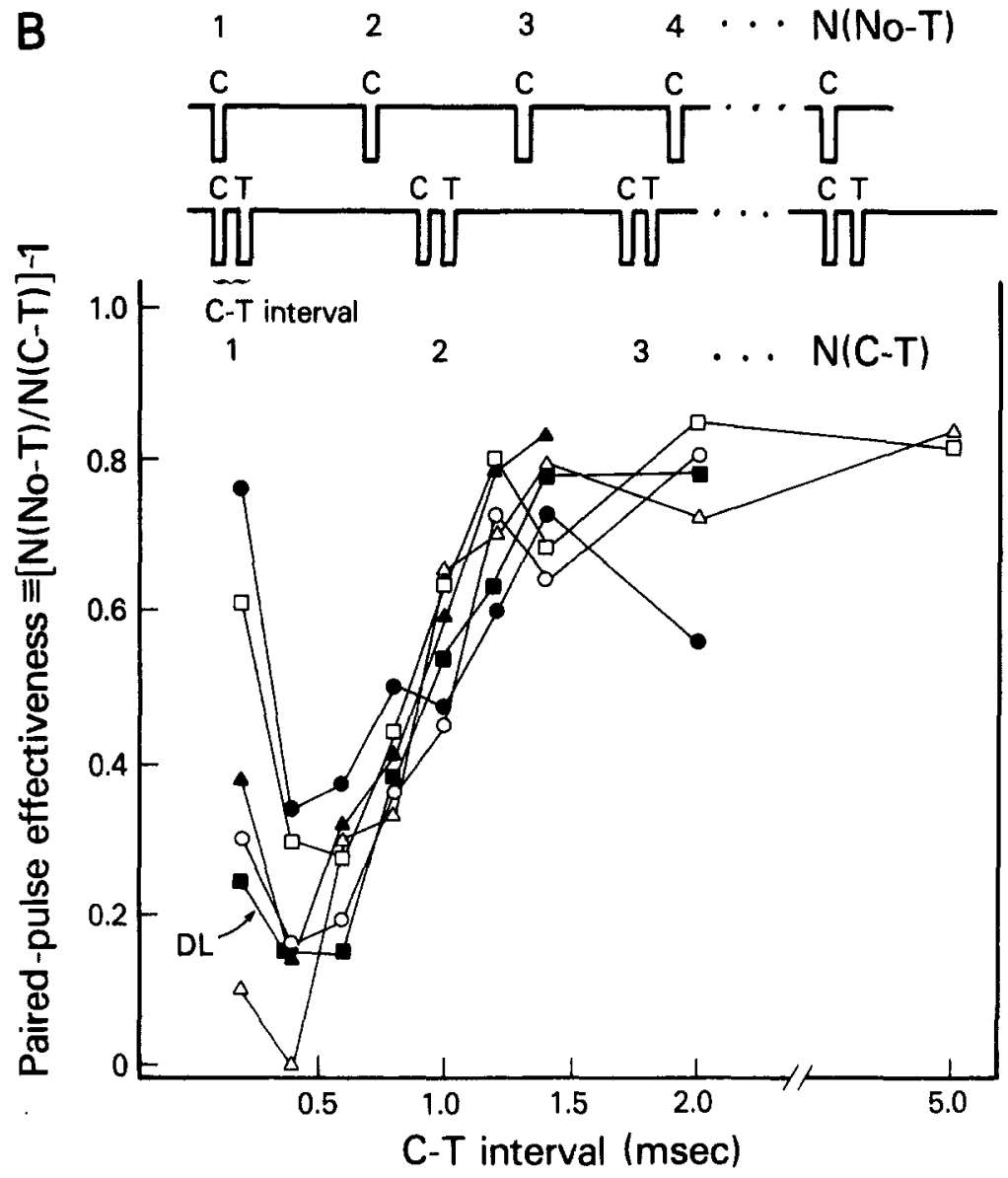
tiveness declines sharply for a very short period of time (about $.5 \mathrm{msec}$ ). Effectiveness then increases sharply for another short period (about .5-1.2 msec). Finally, over a long period $(1.2-5 \mathrm{msec})$, effectiveness rises very little. Deutsch (1964), the first to use pairedpulse experiments to characterize the substrate for self-stimulation, attributed the initial decline in effectiveness to decreasing local potential summation and the later increase in effectiveness to recovery from refractoriness. This two-part interpretation is fundamental to all paired-pulse experiments.

Local potential summation can be expected to occur in axons lying just outside the region within which the $\mathrm{C}$ pulse produces action potentials. In this subliminal fringe, the $\mathrm{C}$ pulse is too weak to fire neurons, but it does depolarize them. If the $T$ pulse is presented before the depolarization decays, its effect will build on the residual. The summated effect of the two pulses fires neurons that neither pulse acting alone can fire. The size of the fringe region in which the summated depolarization exceeds the firing threshold depends on the $\mathrm{C}-\mathrm{T}$ interval and the rate of the decay of the local potentials. When the $T$ pulse comes immediately after the $C$ pulse, it recruits many additional axons. As the $\mathrm{C}-\mathrm{T}$ interval increases, fewer axons are recruited.

Consider now the axons that were fired by the $\mathrm{C}$ pulse; they will be for some time refractory to further excitation. So long as they remain refractory, they will not be fired by the $T$ pulse. The nadir in paired-pulse effectiveness occurs at $\mathrm{C}-\mathrm{T}$ intervals around $.5 \mathrm{msec}$. The $\mathrm{T}$ pulse recruits few additional axons at such a delay, but most of the axons fired by the $\mathrm{C}$ pulse remain refractory and cannot be fired again. As the $\mathrm{C}-\mathrm{T}$ interval increases still further, axons fired by the $\mathrm{C}$ pulse recover from refractoriness. Those that have recovered are fired by the $T$ pulse, which explains the rise in paired-pulse effectiveness beyond C-T intervals of $.5 \mathrm{msec}$.

Work now to be reviewed tests this interpretation and extends it. This work shows that (a) the first phase (local potential summation) is better mapped by trading current against $\mathrm{C}-\mathrm{T}$ interval, whereas the second phase (recovery) is better mapped by trading number of pulse pairs; (b) using pulses of opposite polarity ( $C$ pulse anodal, $T$ pulse cathodal) leads to negative values of pairedpulse effectiveness during the phase of local potential summation; (c) the rate and form of decay of local potential agrees with electrophysiologically obtained values; and (d) the first phase disappears when local summation is prevented by delivering the $\mathrm{C}$ and $T$ pulses on spatially separated electrodes.

The mapping of the poststimulation excitability cycle is extended in four ways: (a) A supernormal period is shown to follow the recovery from refractoriness. (b) The initial rise in effectiveness during recovery is shown to reflect primarily the recovery from absolute refractoriness. (c) The results obtained do not change appreciably when different base frequencies of stimulation are used. (d) Different excitability results are obtained for self-stimulation at other sites and for other behaviors.

Test 1. The choice of an appropriate offsetting variable. Recovery from refractoriness allows the number of firings within a circumscribed region to increase from one to two per pulse pair. Local potential summation, on the other hand, leads to an increased spread of excitation; that is, an increase in the extent of the region in which neurons are fired. The appropriate offsetting variable when number of firings varies is the number of pairs required. The appropriate offsetting variable when the region of suprathreshold excitation varies is the current

Figure 3. Panel A: A family of curves showing bar pressing rate as a function of the number of pairs in a $250 \mathrm{msec}$ train, at $C-T$ intervals ranging from .2 to $2.0 \mathrm{msec}$. (The quantities used to compute the paired-pulse effectiveness are derived from this family by laying a performance criterion across it and dropping the intersections to the abscissa, as illustrated for the quantity N(No-T). [Data from Yeomans, 1975, Figure 2, Animal DL].) Panel B: Paired-pulse effectiveness as a function of C-T interval. (Data from 6 different electrodes in 5 different rats. The cartoon of the stimulating train at the top of this and all subsequent trade-off figures indicates the two parameters traded against one another, in this case $\mathrm{N}(\mathrm{C}-\mathrm{T})$ and the C-T interval. [Adapted from Yeomans, 1975, Figure 4.]) 
intensity. The population of fired neurons is held constant at all $\mathrm{C}-\mathrm{T}$ intervals when the appropriate offsetting variable is used. If the wrong offsetting variable is use, say, when an increased spread of excitation is offset by a reduction in the number of pairs, then the trade-off results will depend on the behavioral importance of the neurons in the newly recruited region. The behavioral importance of the recruitable fringe may vary with, among other things, electrode placement, current intensity, and behavioral effect (Skelton \& Shizgal, 1980). The offsetting variable should always be chosen so as to hold the region of suprathreshold excitation constant.

According to this argument, more variability would be predicted when local potential summation is measured by the required number of pairs than when it is measured by the required current intensity. Deutsch's interpretation, then, predicts low betweenelectrode variability during the initial falling phase when current is the offsetting variable and high between-electrode variability when number of pairs is the offsetting variable.

In Figure 3, Panel B, with the number of pairs as the offsetting variable, the initial falling phase varies considerably from electrode to electrode. Figure 4, Panel A shows the results when current is the offsetting variable (Yeomans, Matthews, Hawkins, Bellman, \& Doppelt, 1979). The low variability between electrodes supports the interpretation that local potential summation, a spatial variable, produces the initial decline in paired-pulse effectiveness.

In Figure 3, Panel B, with the number of pairs as the offsetting variable, there is very little variation from electrode to electrode in the second or rising phase of paired-pulse effectiveness. This suggests that the rising phase does not depend on a variable that alters the spatial extent of the firing field (such as local potential summation) but rather on a variable such as recovery from refractoriness that alters the number of firings per neuron.

Test 2. Pulses of opposite polarity are antagonistic in the first phase but agonistic in the second phase. In this test, by using anodal $C$ pulses and cathodal $T$ pulses, the first phase decline is reversed into a rising phase while the second phase is unchanged.

Anodal $\mathrm{C}$ pulses hyperpolarize axon mem- branes locally, Consequently, more depolarizing current will be required to excite these neurons. Paired-pulse effectiveness will, therefore, be decreased by the anodal $\mathrm{C}$ pulses antagonizing the effects of the cathodal $\mathrm{T}$ pulses at $\mathrm{C}-\mathrm{T}$ intervals during which local potential summation occurs.

Hyperpolarizing entry currents, however, are accompanied by depolarizing exit currents, which, if strong enough, will fire action potentials. The smaller proportion of neurons excited in this way will become refractory to stimulation by the cathodal $T$ pulses. As these axons recover, they will be fired a second time by the cathodal T pulses, so that paired-pulse effectiveness will rise during the second phase, as in previously described experiments.

As may be seen in Figure 4, Panel B, these expectations have been borne out: At C-T intervals less than $.6 \mathrm{msec}, \mathrm{C}$ and $\mathrm{T}$ pulses of opposite polarity act antagonistically (paired-pulse effectiveness is negative). At greater $\mathrm{C}-\mathrm{T}$ intervals, they act agonistically (paired-pulse effectiveness is positive). By comparing Panels A and B in Figure 4, one may estimate the relative contribution of the two phases at any given $\mathrm{C}-\mathrm{T}$ interval. In Panel A, the two effects combine additively; in Panel B, they combine subtractively. This reversal of paired-pulse effectiveness in the first phase shows unambiguously that different mechanisms are involved in the two phases.

Test 3. The behaviorally measured decay of local potential is quantitatively similar to what is found when recording from single units. Using current as the offsetting variable, one can make a fine-grained analysis of the decay of local potentials. In single units in the brain, this decay is approximately exponential (Yeomans et al., 1979). The rate of decay is believed to be determined by the time constant of the membrane (the product of its resistance and its capacitance). The rate of decay is the same following both depolarization and hyperpolarization. Yeomans et al. (1979) found that exponential decay curves with a time constant of about $.1 \mathrm{msec}$ fit the data in Figure 4 over intervals from .05 to $.3 \mathrm{msec}$. The deviations from exponential decay at intervals beyond $.3 \mathrm{msec}$ suggest the existence of some neurons that have slower decay rates.

Test 4. The initial phase is absent when 


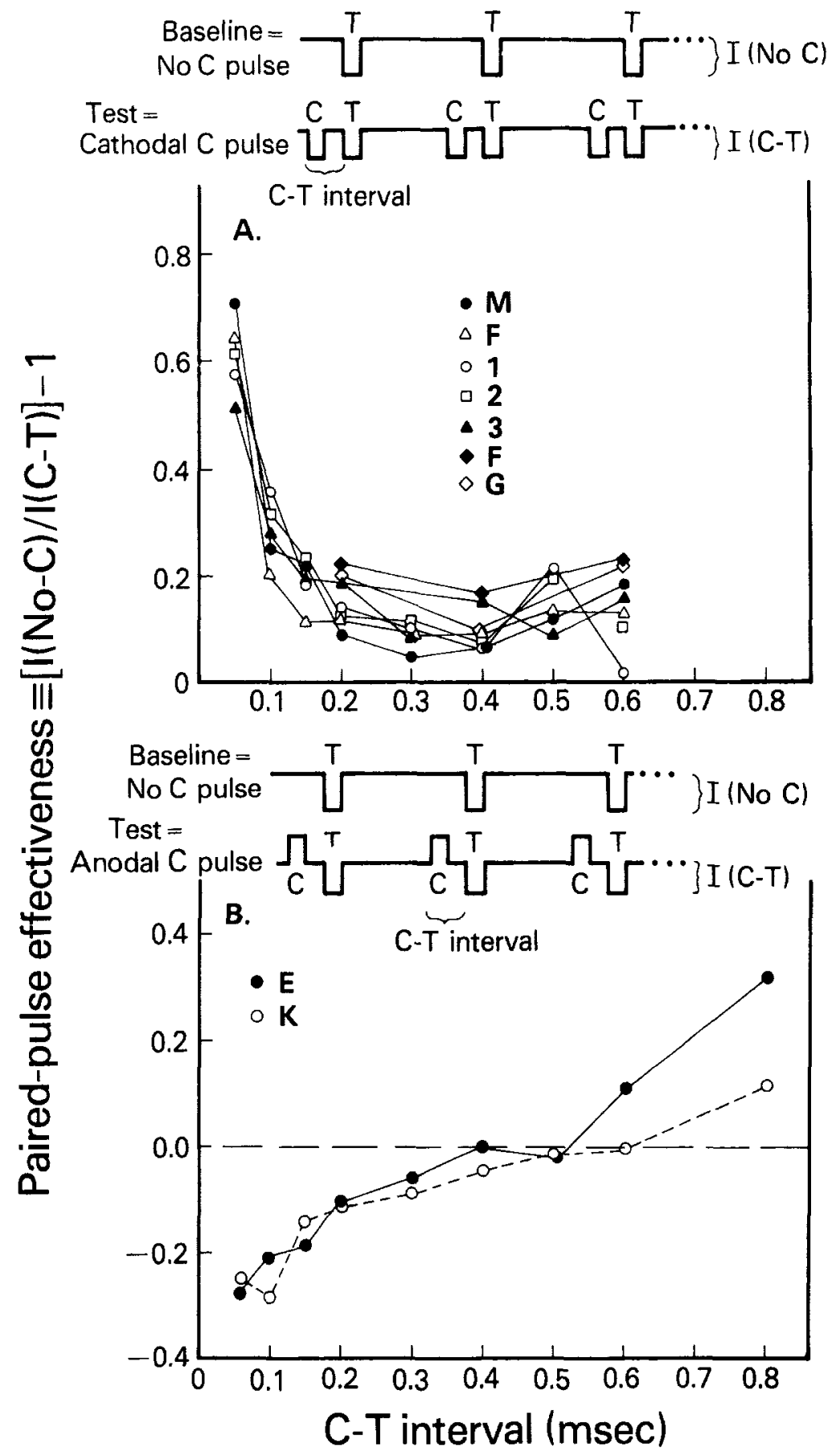

Figure 4. Panel A: Paired-pulse effectiveness over C-T intervals of .05-.6 msec, as measured by varying current intensity. (Note the close agreement in data from different electrodes. When the $C$ pulse is cathodal, paired-pulse effectiveness is positive over the range of local potential summation, that is, the effects of $\mathrm{C}$ and $\mathrm{T}$ pulses summate. [Data originally published by Yeomans et al., 1979, rats $\mathrm{M}, \mathrm{F}, 1$, 2. \& 3; Yeomans \& Davis, 1975, rats F \& G] Panel B: Paired-pulse effectiveness over C-T intervals from .05 to $.8 \mathrm{msec}$ when the $\mathrm{C}$ pulse is anodal. (In the range of local potential summation, the effects of anodal $C$ pulses and cathodal $T$ pulses cancel, so paired-pulse effectiveness is negative. At $C-T$ intervals beyond $.6 \mathrm{msec}$, in the range where recovery from refractoriness dominates, the effects summate, and paired-pulse effectiveness is positive. [Data from Yeomans et al., 1979.]) 
$C$ and $T$ pulses are delivered via separated electrodes, whereas the second phase is present but delayed. If the first phase of the curve in Figure 3, Panel B reflects local potential summation, then it should not be seen when the $C$ and $T$ pulses are delivered via different electrodes spaced some distance apart along the cable, because the subthreshold depolarization left by the $C$ pulse is a local nonconducted change, that is, a change not seen any distance away. For reasons explained below, however, the recovery from refractoriness should be present in the twoelectrode experiment but delayed (Shizgal, Bielajew, Corbett, Skelton, \& Yeomans, 1980). A look ahead at Figure 7 reveals that both these expectations are borne out by the data.

Extension 1. A period of supernormal excitability is revealed when $T$ pulses are weaker than $C$ pulses. In some central axons, there is an unusually pronounced period of supernormal excitability following the period of relative refractoriness (Swadlow \& Waxman, 1976). This supernormal period should be manifested in experiments where the $\mathrm{T}$ pulse is weaker than the $\mathrm{C}$ pulse, but not in experiments where the two pulses are of equal strength.

A $T$ pulse that is weaker than a $C$ pulse normally fires fewer axons. There is, therefore, a reservoir of axons that are fired by the $C$ but not the $T$ pulse. If a period of supernormal excitability supervenes in these axons following the recovery from refractoriness, the weak $T$ pulse will be able to fire some fraction of this reservoir. In other words, during the supernormal period, weak $T$ pulses will be unusually effective. This unusual effectiveness, however, depends on the $T$ pulses' being weaker than the $\mathrm{C}$ pulses. When $\mathrm{T}$ pulses are as strong or stronger than $C$ pulses, there is no reservoir of axons that are normally fired by the $C$ pulse but not the $T$.

Figure 5 shows paired-pulse effectiveness as a function of $\mathrm{C}-\mathrm{T}$ interval under three conditions: $T$ pulses weaker then, equal to, or stronger than $\mathrm{C}$ pulses. Note than in the "weaker than" condition, paired-pulse effectiveness is significantly elevated over C-T intervals of 1.2-5.0 msec, the intervals immediately following the recovery from re- fractoriness. All other $\mathrm{C}-\mathrm{T}$ intervals yield the same relative paired-pulse effectiveness across all three conditions. The neurons mediating MFB self-stimulation, therefore, have a supernormal period.

Extension 2. Separating absolute and relative refractory periods. The contributions of absolute refractory periods can be separated from the contributions of relative refractory periods using $\mathrm{C}$ and $\mathrm{T}$ pulses of unequal strength. If the $T$ pulses are made more intense than the $C$ pulses, the contribution of relative refractoriness to the recovery curve should be considerably reduced (Yeomans, 1979). Each neuron excited by a $C$ pulse will be stimulated by a more intense $T$ pulse and should thus be fired again at a shorter $\mathrm{C}-\mathrm{T}$ interval after the absolute refractory period. At the placement that yielded the data in Figure 5, reliable differences were not found between the conditions where the $T$ pulse was either equal to or stronger than the $C$ pulse. This suggests that relative refractory period contributions at this site were small and that the rise of excitability in the refractory period range can be attributed predominantly to the recovery of more and more neurons from their periods of absolute refractoriness. Thus, we can specify the relative contribution of neurons with different absolute refractory periods to MFB self-stimulation (see percentages below). At other sites, there do appear to be appreciable contributions from relative refractoriness (Jordan, Bielajew, \& Shizgal, Note 1).

In summary, by varying the relative intensities and the polarity of $\mathrm{C}$ and $\mathrm{T}$ pulses, one may estimate the contributions of four axonal factors: local potential summation, supernormal periods, absolute refractory periods, and relative refractory periods. Reversing the polarity of $\mathrm{C}$ pulses subtracts local potential summation contributions from refractory period contributions. Increasing the intensity of $C$ pulses reveals a supernormal period. Increasing the intensity of $T$ pulses indicates that the recovery from relative refractoriness is not an important factor. Among axonal excitability factors, only absolute refractory period contributions can account for the sharp rise in paired-pulse 

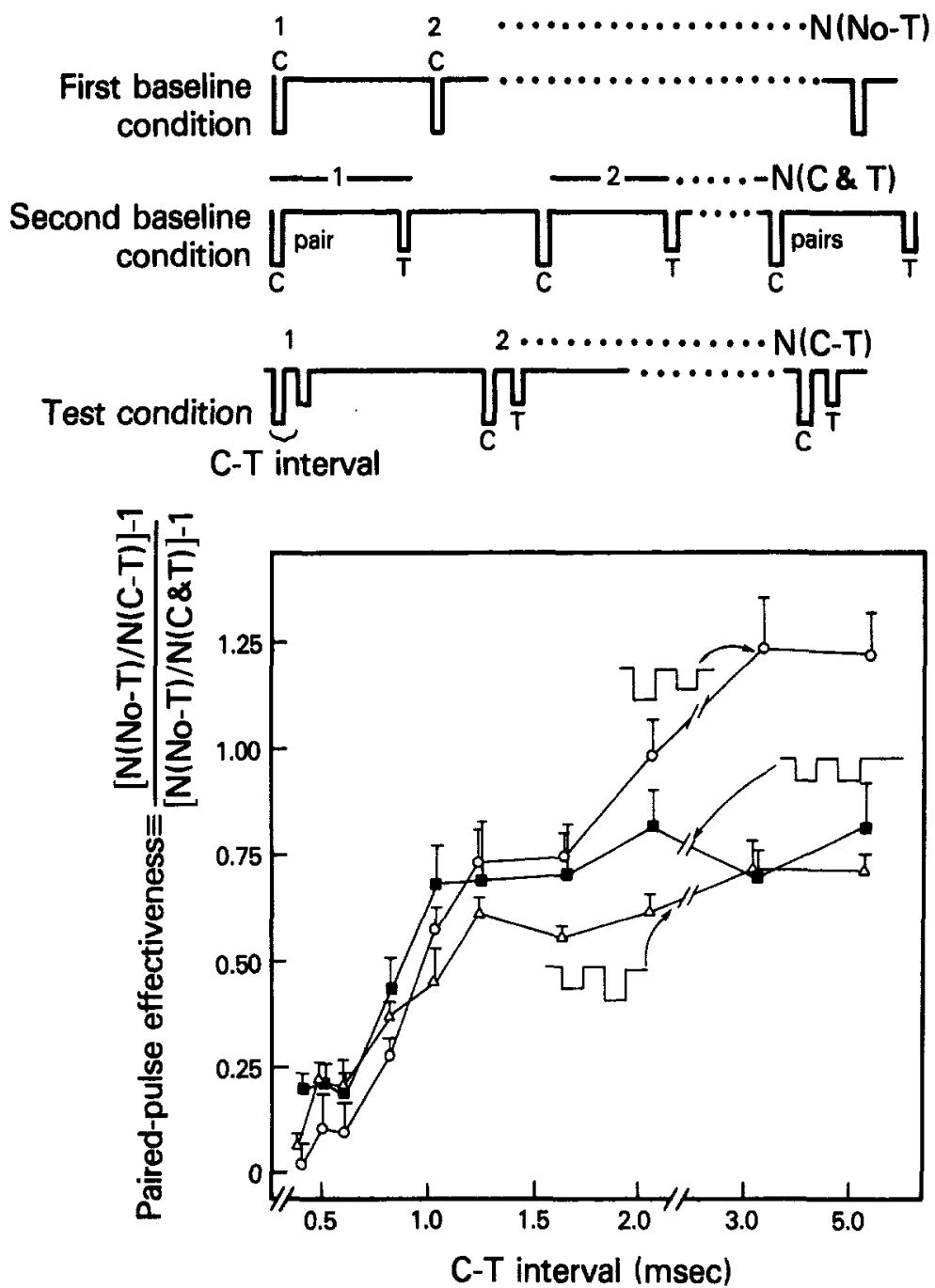

Figure 5. Paired-pulse effectiveness as a function of $\mathrm{C}-\mathrm{T}$ interval when the $\mathrm{T}$ pulse is either less than, equal to, or greater than the $C$ pulse. (When $C$ and $T$ pulses are unequal, the formula for paired-pulse effectiveness requires a second baseline condition, in which the unequal $C$ and $T$ pulses are delivered evenly spaced [see Yeomans, 1979, for rationale]. The vertical bars indicate 1 standard error of the mean. Note that when the $T$ pulse is weaker than the $C$, one sees evidence of a supernormal period. [Data from Yeomans, 1979.])

effectiveness when equal current pulses are used.

Extension 3. The paired-pulse effectiveness function does not depend on the base frequency. Hawkins, Roll, Puerto, and Yeomans, (Note 2) tested the robustness of the number-of-pairs scale using a y-maze procedure. On each trial each rat was given a priming dose of five trains of $100-\mathrm{Hz}$ brain stimulation in the start arm, then allowed to choose one of the two goal arms. The choice of one arm yielded a reference train of evenly spaced pulses. The choice of the other yielded a train of paired pulses. Trials were run until it was determined how many paired pulses at a given C--T interval were 
required to equal the reward provided by the reference train. The range over which the number of pulse pairs in the test train varied during the course of a refractory period determination depended on the choice of a pulse frequency for the .5 -sec reference train. Five different pulse frequencies were used: $25,50,100,150$, and $200 \mathrm{~Hz}$; that is, the reference train had $13,25,50,75$, or 100 pulses in it. When the reference train had 13 pulses, the number of pulse pairs in the .5 -sec test train ranged from about 6 to about 13 , depending on the $\mathrm{C}-\mathrm{T}$ interval. In other words, the pair frequency ranged from 12 to 26 pairs per second. When the reference train had 100 pulses, pair frequency in the test train ranged from 50 to $100 / \mathrm{sec}$. The values for paired pulse effectiveness at each C- $T$ interval were similar across these different ranges of variation in the number of pulse pairs (different ranges of pair frequency).

This experiment makes two points. First, the paired-pulse effectiveness depends very little, if at all, on the number of pulses delivered in a train. This supports the use of the number of pulses as a scale to measure paired-pulse effectiveness. The one qualifier to this conclusion is that the local potential summation phase was found to be more sensitive to the choice of frequency than the refractory period phase of the curve.

The second point is that one obtains very similar estimates of the quantitative properties of the underlying substrate using quite different tasks. The estimates of axonal excitability as a function of $\mathrm{C}-\mathrm{T}$ interval are the same whether one uses a bar-pressing task or a preference task.

Different courses of recovery at other sites and for other behavioral effects of stimulation. Dennis, Yeomans, and Deutsch (1976) studied the recovery from refractoriness in the substrate for the aversive effects of stimulating the medial lemniscus. They found more abrupt recovery than is found for rewarding effects. Bielajew, Jordan, Ferme-Enwright, and Shizgal (in press) studied the recovery from refractoriness in the substrate for self-stimulation of the periaqueductal grey and found a slower recovery than is found at MFB sites. Considerably delayed recovery has been found at frontal cortex sites of self-stimulation (Schenk, Shizgal, \& Bielajew 1980). Miliaresis and Rompré (1980) studied the recovery from refractoriness in the substrate for the turning induced by stimulation of the median raphe. The recovery here was more abrupt than for MFB self-stimulation.

Each of these studies traded the number of pulse pairs against $\mathrm{C}-\mathrm{T}$ interval and so should be distinguished from the many studies that have obtained different "refractory periods" without using trade-off procedures or without using the number of pulse pairs as the offsetting variable (see Yeomans, 1975, for discussion). These studies show that the neural substrates for the differing behavioral effects of brain stimulation often have different refractory periods and that self-stimulation at periaqueductal grey and frontal cortex sites is mediated by neural substrates that differ in their refractory periods from the substrate for self-stimulation of the MFB and ventral tegmentum.

Why only first-stage properties are measured. We have assumed that paired-pulse effectiveness at $\mathrm{C}-\mathrm{T}$ intervals from .1 to 5.0 msec depends entirely on the properties of the first stage axons. Why could one not assume that some or all of the recovery from refractoriness or the subsequent supernormal period is due to properties of the second stage? Gallistel (1973) points out that the temporal characteristics of most synapses are such that differences on the order of tenths of milliseconds in the temporal separation of presynaptic impulses would not observably affect excitability at the synapse. Recent electrophysiological evidence indicates that these differences may disappear before a pair of spikes even reaches the first synapse. Kocsis, Swadlow, Waxman, and Brill (1979), recording from long, small-diameter myelinated axons in the rabbit visual system, showed that the conduction velocity of a second action potential was reduced if it was initiated during the period of relative refractoriness and increased if it was initiated during the supernormal period. The result was that for $\mathrm{C}-\mathrm{T}$ intervals between .9 and $2.0 \mathrm{msec}$ the temporal separation of the two spikes by the time they arrived at the 
recording electrode was always the same (Figure 6). The second stage, then, will see no differences between $\mathrm{C}-\mathrm{T}$ intervals in the relative refractory or supernormal periods when the conduction distance is long.

Conclusions. From paired-pulse studies with single electrodes we draw the following conclusions about the properties of the reward-relevant MFB axons excited by the electrode current.

1. Local potentials decay very rapidly, with a time constant of about .1 msec. Unfortunately, little is known about local potential properties of MFB units beyond the three observed by Yeomans et al. (1979). Those three units had almost identical decay.

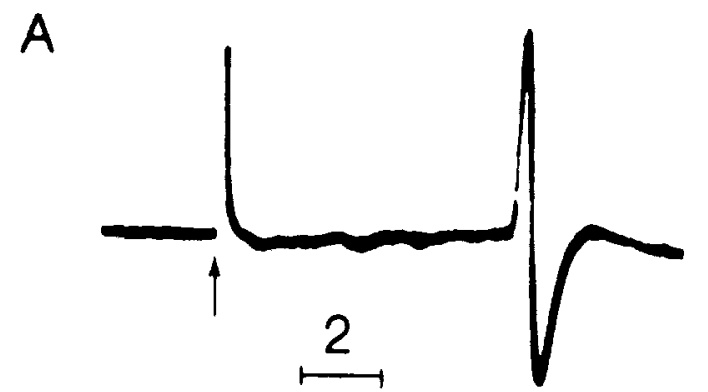

msec

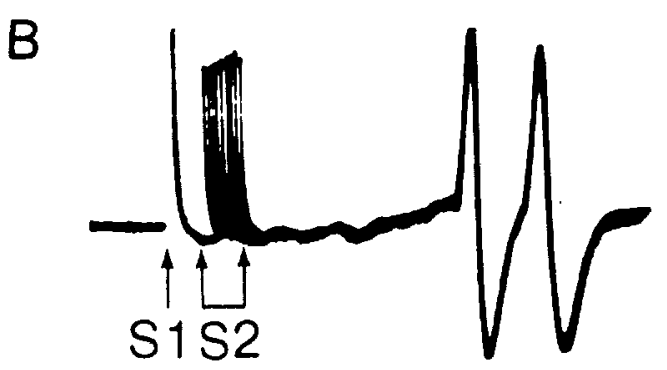

Figure 6. Recording of antidromic spikes evoked by thalamic stimulating pulses in an axon efferent from the rabbit visual cortex. (The recording electrode was about $15 \mathrm{~mm}$ distant from the stimulating electrode.) Panel A: A control response. (The arrow indicates the electrical artifact from the stimulating pulse. The action potentials follow this at a latency of $7.7 \mathrm{msec}$; therefore, conduction velocity $=1.9 \mathrm{~m} / \mathrm{sec}$.) Panel B: Paired pulses with $\mathrm{C}-\mathrm{T}$ intervals from .9 to $2.0 \mathrm{msec}$. (The $\mathrm{C}$ pulse was triggered at a fixed point for all sweeps; the artifact from it is labeled $\mathrm{S} 1$. The variable $\mathrm{C}-\mathrm{T}$ interval is manifest in the smear of T-pulse artifacts, labeled S2. Note that despite the variation in $\mathrm{C}-\mathrm{T}$ interval, the second spike follows the first at an unvarying latency of about $1.7 \mathrm{msec}$. [From Kocsis et al., 1979. Published by permission of Academic Press and the authors.])
2. The distribution of absolute refractory periods of directly stimulated, reward relevant MFB neurons is of the following type: About $15 \%$ of the rewarding signal is produced by axons with absolute refractory periods from .4 to $.6 \mathrm{msec}$; about $25 \%$ from .6 to .8 ; about $20 \%$ from .8 to 1.0 ; and about $15 \%$ from 1.0 to 1.2 . This distribution overlaps with refractory periods obtained from units directly driven by rewarding MFB electrodes (Rolls, 1975; see also below). These percentages do not specify the relative numbers of axons in each category, since a large number of contributing axons might have a miniscule effect on behavior. The result, though, specifies the relative importance to the behavior in question of axons with one or another refractory period. This ability to measure the importance of neurons to the resulting behavior is a major contribution of this technique.

3. We are uncertain how to account for the remaining $10 \%-25 \%$ increase in pairedpulse effectiveness, which occurs gradually over C-T intervals from 1.2 to $15 \mathrm{msec}$. It is probable that some of this is due to the absolute refractory periods of slower recovering axons. Perhaps these unusually long refractory periods are an artifact of the very intense currents to which axons close to the electrode are subjected (see below, the discussion of the differing rates of recovery in one-electrode vs. two-electrode experiments). At these longer intervals, slower synaptic phenomena may also make some contribution.

4. The axons have a supernormal period.

\section{Two-Electrode Experiments}

In the experiments just described, pairs of stimulation pulses are applied to a single electrode and the $\mathrm{C}-\mathrm{T}$ interval is varied. A modification of this method, which involves the use of two electrodes, is designed to determine whether different self-stimulation sites are connected by a common cable. In cases where reward-relevant fibers directly link two self-stimulation sites, the two-electrode paired-pulse technique provides an estimate of the conduction velocity in these fibers. Since conduction velocity and refractory period are related attributes, the results 
of one- and two-electrode paired-pulse experiments provide convergent characterizations of the substrate for self-stimulation.

The two-electrode paired-pulse technique was first used by Lucas (1913) to study the effects of alcohol on the conduction velocity in nerve. The rationale rests on the fact that when two action potentials approach each other from opposite directions and collide, neither propogates past the point of collision. Although action potentials in an intact nervous system generally travel only toward the synaptic terminals, electrical stimulation triggers an action potential that travels in the normal direction (orthodromic) and an action potential that travels away from the stimulation site toward the soma (antidromic). When an axon is stimulated at two different sites, the antidromic action potential triggered by the electrode closer to the terminals ("downstream") collides with the orthodromic action potential triggered by the electrode closer to the soma ("upstream"). Only the orthodromic action potential triggered by the downstream electrode reaches the terminals. This blocking effect disappears if the pulse on the upstream electrode is delayed long enough to allow the antidromic action potential from the downstream electrode and its trailing refractory zone to propogate past the upstream site. The required delay, subsequently referred to as the collision interval, is the sum of the time required for conduction between the two sites and the refractory period.

Lucas used the one-electrode set-up to measure the refractory period and the twoelectrode set-up to measure the collision interval. To get the conduction time between the two electrodes, he subtracted the refractory period (single electrode) from the collision interval (double electrode). To get conduction velocity, he divided the conduction time by the distance between the electrodes.

Lucas performed his conduction velocity measurements on a nerve-muscle preparation, where the anatomical linkage of the two stimulation sites was known to consist of a parallel bundle of axons. In applying his method to studying the substrate for selfstimulation, we have turned his reasoning around. We use the two-electrode pairedpulse technique to infer whether a common bundle of reward-relevant axons links the two stimulation sites.

Collision effects in MFB self-stimulation. As early as 1956 , Olds suspected that the trajectory of many reward related neurons followed that of the medial forebrain bundle (MFB). This is a heterogeneous pathway with many sources and projections. Despite 20 years of active work on the problem, there has not yet been a convincing demonstration of which directly stimulated MFB elements subserve self-stimulation. During the past decade, various long-axon, catecholaminergic components of the MFB have been suggested to serve this role. Shizgal et al. (1980) reasoned that if such longaxon neurons were in fact the directly stimulated substrate for MFB self-stimulation, then it siould be possible to demonstrate collision effects when two ipsilateral MFB self-stimulation sites were stimulated concurrently.

The method used by Shizgal et al. closely resembled that used by Yeomans in the experiments described above. The principal modification was that the $C$ pulses were applied to one electrode and the $T$ pulses to the other. As in Yeomans' experiments, the effectiveness of the stimulation at a given $\mathrm{C}$ $T$ interval was scaled in terms of the number of pulse pairs required to produce half of the maximal rate of responding.

The results in Figure 7, Panel $\mathrm{A}$ are from a subject that self-stimulated via ipsilateral electrodes in the lateral hypothalamus and ventral tegmentum. Among the differences between these results and those of one-electrode paired-pulse experiments is the C-T interval at which the increase in the effectiveness of stimulation occurs; the rise occurs later in the two-electrode experiments. In Lucas's (1913) experiment, the C-T interval at which paired-pulse stimulation became fully effective was also longer in the twoelectrode case. Lucas attributed this difference to the time required for conduction between the two stimulation sites. We think the same explanation holds for the MFB self-stimulation data in Figure 7, Panel A.

Several additional manipulations were performed to test this interpretation of the two-electrode results. To derive the solid curve in Figure 7, Panel A, C pulses were 


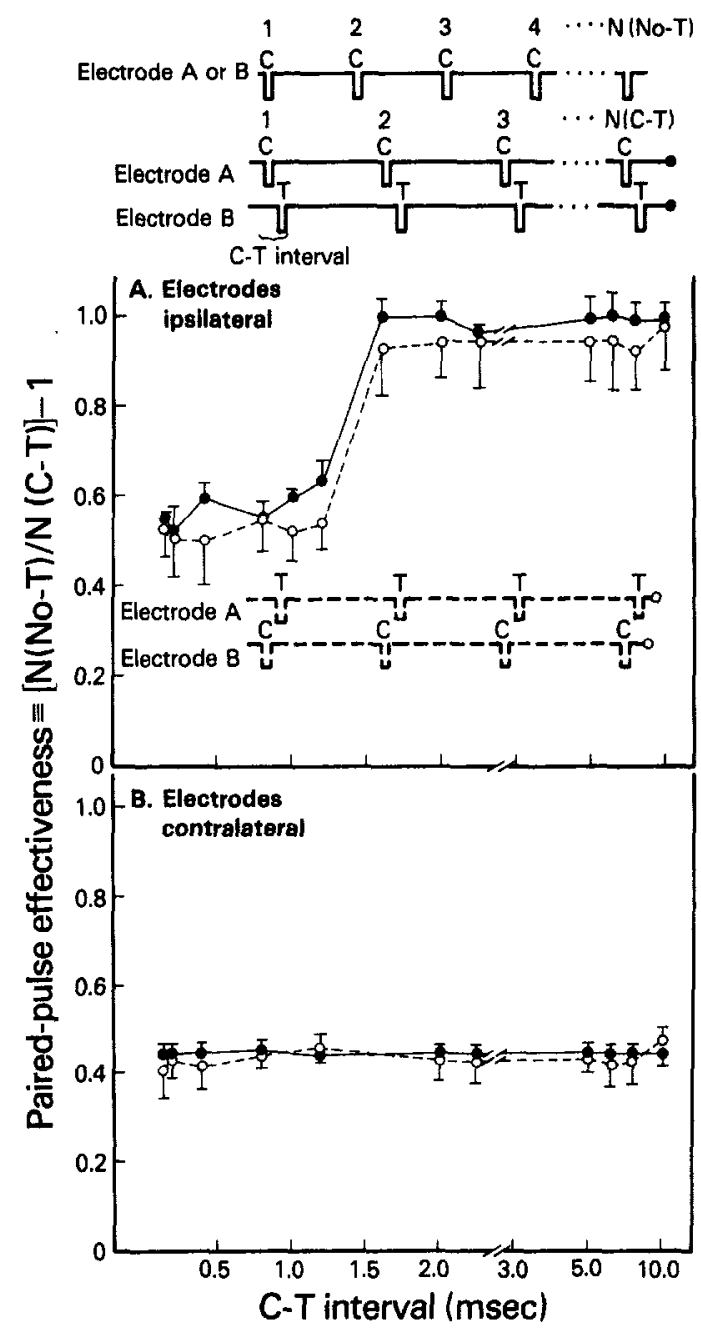

Figure 7. Panel A: Paired-pulse effectiveness as a function of $C-T$ interval when the $C$ and $T$ pulses are delivered via separate electrodes arrayed ipsilaterally, one in the lateral hypothalamus and one in the tegmentum. (The baseline, N(No-T), is an average of the number of pulses required when either electrode alone gets evenly spaced pulses.) Panel B: Same experiment as in Panel A but with contralateral electrodes. (Data from Schizgal et al., 1980.)

applied to the hypothalamic electrode and $T$ pulses to the tegmental electrode. When this arrangement was reversed, there was no change in the effect of varying the $C-T$ interval (dashed curve in Figure 7, Panel A). It is difficult to imagine a synaptic linkage between two stimulation sites that would permit a steplike change such as that seen in Figure 7, Panel A. It is even more difficult to conceive of a synaptic linkage that would be indifferent to the order in which the paired pulses are applied.

The similarity of the two curves in Figure 7, Panel $A$ is to be expected if the two sites are directly linked by reward-relevant axons. Irrespective of whether the $C$ pulse is applied via the upstream or the downstream electrode, the collision block is due to the antidromic action potential triggered by the downstream electrode. When the $\mathrm{C}$ pulse is applied via the upstream electrode, the collision interval equals the sum of the interelectrode conduction time and the refractory period under the downstream electrode; when the $C$ pulse is applied via the downstream electrode, the collision interval equals the sum of the same conduction time and the refractory period under the upstream electrode. The collision interval should thus be the same in both cases, provided that the two refractory periods are also the same, which they are. Figure 8 shows that the courses of recovery at the two sites are identical. (As might be expected, the magnitude of local potential summation varies at the two sites.)

If the results in Figure 7, Panel $A$ are correctly interpreted as collision effects, then one should not see such results when the linkage between the two stimulation sites is synaptic. Since no direct axonal connections have been demonstrated between the two contralateral hypothalamic nuclei, Shizgal et al. (1980) chose bilateral, lateral hypothalamic stimulation as a test case. The results of this two-electrode paired-pulse experiment are shown in Figure 7, Panel B. Unlike the unilateral results, these curves are flat; there is no steplike rise. This finding was confirmed in three additional subjects. Thus, the abrupt rise in the unilateral results must be attributed to some feature of the anatomical linkage between the ipsilateral stimulation sites that was not present in the bilateral case.

That this feature was, in fact, a bundle of directly stimulated, reward-related axons linking the two ipsilateral stimulation sites is supported by an additional set of data. Not all of the results of the unilateral two-electrode experiment resemble those in Figure 7, Panel A. In fact, some curves look like those obtained from subjects with bilateral 


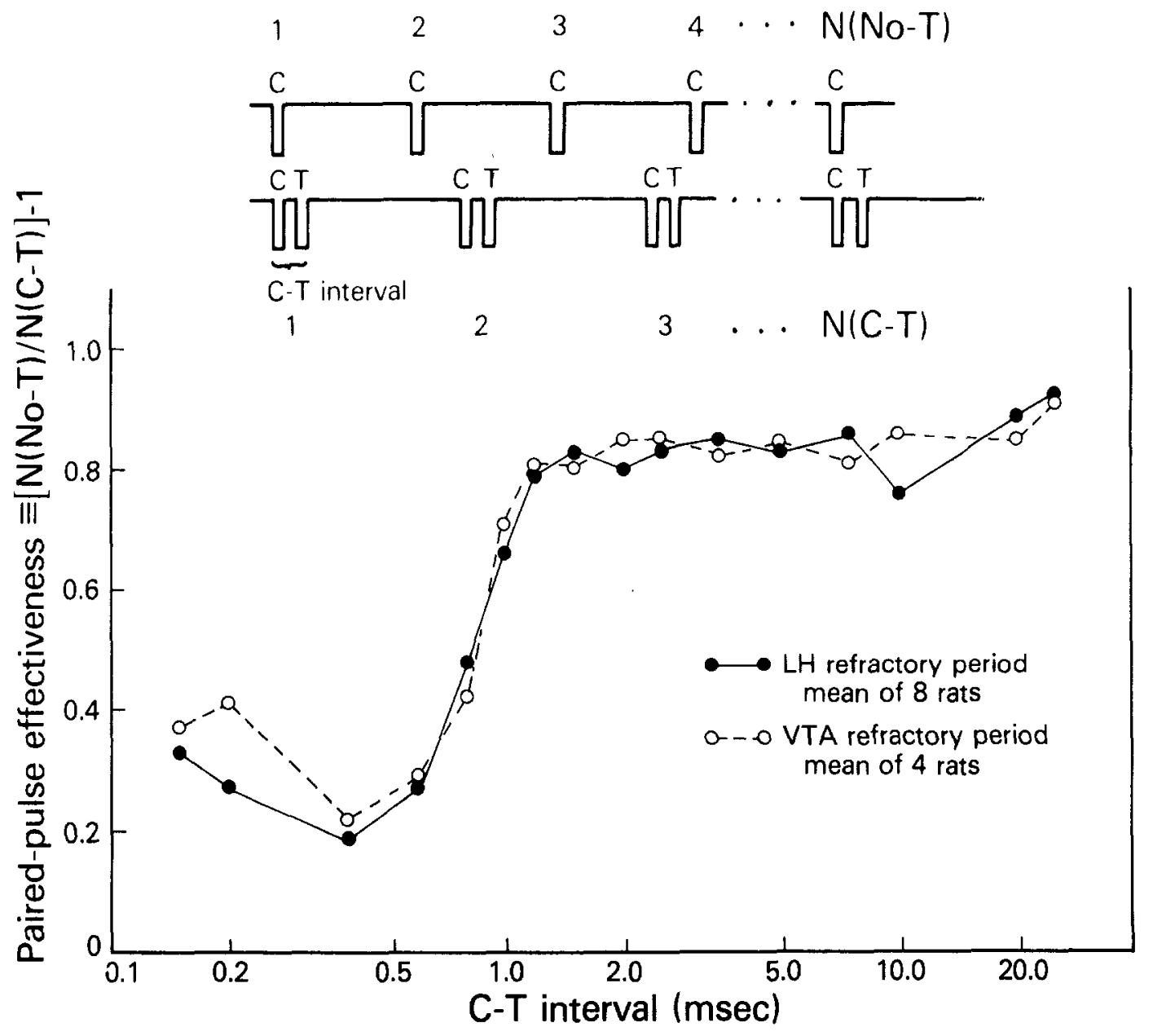

Figure 8. Paired-pulse effectiveness as a function of $\mathrm{C}-\mathrm{T}$ interval at two different sites along the MFB-the lateral hypothalamus (LH) and the ventral tegmentum (VTA). (Data compiled from Shizgal et al., 1980, and Shizgal, Bielajew, \& Yeomans, 1979.)

electrodes. Shizgal et al. proposed that such results would be expected if the two unilateral stimulation fields were misaligned, that is, if they stimulated different elements within the same bundle of fibers (Figure 9). To evaluate this hypothesis, they increased the current intensities and retested a subject with unilateral electrodes that had manifested no collision-like effect when lower currents had been used. Whereas the low current results resembled the flat curves in Figure 7, Panel B, the curves obtained at the higher currents resembled the step-like curves in Figure 7, Panel A. Raising the stimulation currents increases the size of the effective stimulation fields, which, as illustrated in Figure 8, should improve the odds that both fields will activate a given fiber. This finding is thus consistent with the idea that collision effects are responsible for the steplike functions.

Estimates of conduction velocity and fiber diameter. There are several reasons for attempting to extract estimates of conduction velocity from the unilateral two-electrode results. First, these estimates and the refractory period estimates from one-electrode studies provide a mutual check. The quantitative relationship between these characteristics that has been established 


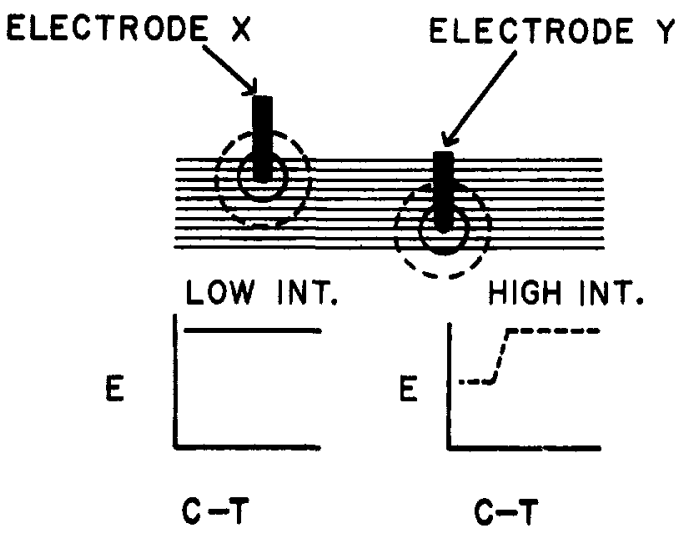

Figure 9. Schematic illustration of why the existence of a collision effect may depend on the intensities of the stimulating currents. (The solid circles surrounding the two electrode tips represent the regions of effective excitation at low intensities. The two regions do not have any axons [horizontal lines] in common, hence there is no collision effect [flat graph, lower left]. Raising the current intensities expands the regions of effective excitation so that they have axons in common [dashed circles]. The result is a collision effect [steplike graph, lower right]. Abbreviations: $\mathbf{E}=$ paired-pulse effectiveness; $C-T=C-T$ interval. [From Shizgal et al., 1980.])

both for peripheral and for central neurons ought to be obeyed by the behaviorally derived estimates. Second, conduction velocity also predicts fiber diameter. The plausibility of these estimates can be further checked by referring to anatomical studies of the stimulation sites. If the estimates are valid, fibers of the predicted diameters should be present at the stimulation sites. As explained below, this comparison also provides a clue as to whether the directly stimulated, reward-related fibers are myelinated. Third, the behaviorally derived conduction velocity estimates can be compared with the physiologically measured conduction velocities of various MFB fibers. Only fibers with conduction velocities within or near the range of the behavioral estimates can be considered as likely components of the substrate for the collision effects in the two-electrode results.

As regards the first point, the conduction velocity estimates that Shizgal et al. derived from their results ranged from 1.8 to $8.0 \mathrm{~m} /$ sec. Recovery from refractoriness in Yeomans' self-stimulation data begins at .4-.6 msec and is $70 \%-80 \%$ complete by $1.2 \mathrm{msec}$. Swadlow and Waxman (1976) found that axons in the corpus callosum with conduction velocities similar to those reported by Shizgal et al. had refractory periods of .7$1.4 \mathrm{msec}$. Thus, the relationship between behaviorally derived estimates of conduction velocity and refractory period in reward related MFB neurons is similar to the relationship between these properties that is observed in electrophysiological studies of other central axons.

As regards the second point, the classic relationship between conduction velocity and fiber diameter (Hursh, 1939) was based on studies of peripheral nerve. Recently, Waxman and his colleagues (Waxman \& Bennett, 1972; Waxman \& Swadlow, 1976; Swadlow \& Waxman, 1976) have extended this analysis to central axons. Working with both myelinated and unmyelinated fibers in the splenium of the rabbit corpus callosum, they found that the match between the ranges and distributions of conduction velocities and fiber diameters was compatible with the relationships established for peripheral fibers. Waxman and Bennett's equations for peripheral axons can then be used to predict the diameter of directly stimulated reward-related fibers from Shizgal et al.'s conduction velocity estimates. If we assume the fibers to be unmyelinated, the estimates of their diameter range from .7 to $13.3 \mu$. If we assume the fibers to be myelinated, the estimates range from .3 to $1.5 \mu$.

The estimates for unmyelinated fibers are implausible; that is, unmyelinated fibers of the very large diameters required to match most of the conduction velocity estimates have not, to our knowledge, been found in the central nervous system. In contrast, the estimates for myelinated fibers are compatible with the actual fiber spectrum of the lateral hypothalamus. Szabo, Lénard, and Kosaras (1974) determined that the great majority of myelinated lateral hypothalamic axons were between .5 and $2.0 \mu$ in diameter.

Differences in rate of recovery. The recovery from refractoriness is more abrupt in the two-electrode data than in the one-electrode data. If the collision interval were the simple sum of a conduction time and the same refractory period as that measured in the one-electrode experiments, then no such difference should exist. We can think of two 
interpretations of this difference. The first interpretation recognizes that the reward cable contains axons with differing absolute refractory periods. Yeoman's absolute refractory period determinations argue that the behaviorally derived excitability curves for self-stimulation reflect a weighted combination of the refractory period characteristics of a mixed population of neurons. The neurons passing through both fields in the two-electrode experiments may be only a portion of this population-a portion with more homogeneous refractory periods. This smaller variance may be reflected in the steeper rate of recovery. The collision block in Shizgal et al.'s experiment was never complete: that is, the values for paired-pulse effectiveness at the short $C-T$ intervals were always non-zero. Thus, the reward-related fibers linking the two stimulation sites were only a portion of the total population of reward-related fibers directly stimulated at each site.

Alternatively, the difference in the rate of recovery observed in the one- and two-electrode experiments may stem from differences in the causes of refractoriness. In the one-electrode case, the axons are recovering from the effects of an artificial stimulating field. The refractory period that contributes to the collision interval is the naturally occurring one that follows in the wake of a propagating action potential. The artificial stimulating field simultaneously excites many nodes, whereas a naturally propagating action potential sequentially activates successive nodes. In addition, the artificial field will be unnaturally intense for many axons. These abnormalities may distort the relationships between transmembrane voltage, time and ionic conductances and thereby change the time course of recovery.

Electrophysiological data. The role of behavioral trade-off data in the process of identifying the substrate for the behavior is to guide the evaluation of electrophysiological and neuroanatomical data. In the present instance, the data from the paired-pulse experiments enable us to use electrophysiological data to reject the hypothesis that catecholaminergic neurons constitute the firststage neurons in electrical self-stimulation of the brain. The electrophysiologically mea- sured conduction velocities for catecholaminergic fibers $(.3-1.5 \mathrm{~m} / \mathrm{sec})$ are too slow and the refractory periods $(1.8-20 \mathrm{msec})$ too long to account for the results of pairedpulse experiments (see Faiers \& Mogenson, 1976; Feltz \& Albe-Fessard, 1972; German, Dalsass, \& Kiser, 1980; Guyenet \& Aghajanian, 1978; Segal \& Bloom, 1974; Takigawa \& Mogenson, 1977). The conclusion that the directly stimulated axons are not dopaminergic is somewhat surprising. The very close correlation between successful self-stimulation sites and the locus of the dopaminergic projections in the ventral tegmentum and MFB (Corbett \& Wise, 1980) and the extensive pharmacological data implicating dopamine in self-stimulation (Wise, 1978) favor the hypothesis that self-stimulation at the sites we are here concerned with is mediated by the direct excitation of the dopaminergic projection. Except for the data we have just reviewed, we know of no strong evidence against this hypothesis.

Paired-pulse data do not rule out a role for catecholaminergic fibers in MFB selfstimulation. Such fibers could conceivably contribute to the later phase of recovery from refractoriness and, if stimulated by only one of each electrode pair, may account for the incompleteness of the collision block. Perhaps even more likely is the possibility that the high thresholds (Yim \& Mogenson, 1980; German et al., 1980) of these fibers prevent their activation at the pulse width and current intensity combinations used in self-stimulation experiments, but that they are transsynaptically activated. In other words, one way to reconcile our data with the pharmacological data implicating catecholamine neurons is to assume that the catecholamine neurons comprise part or all of the second-stage network. Another possibility is that catecholamine neurons do not form part of either stage; that is, that they do not carry the rewarding signal, but rather supply some, possibly tonic, input without which the rewarding signal cannot be converted into an engram.

As we have emphasized, the paired-pulse data indicate that the first-stage neurons are small-diameter myelinated axons. It has long been known on the basis of anatomical methods that such axons constitute a sizable 

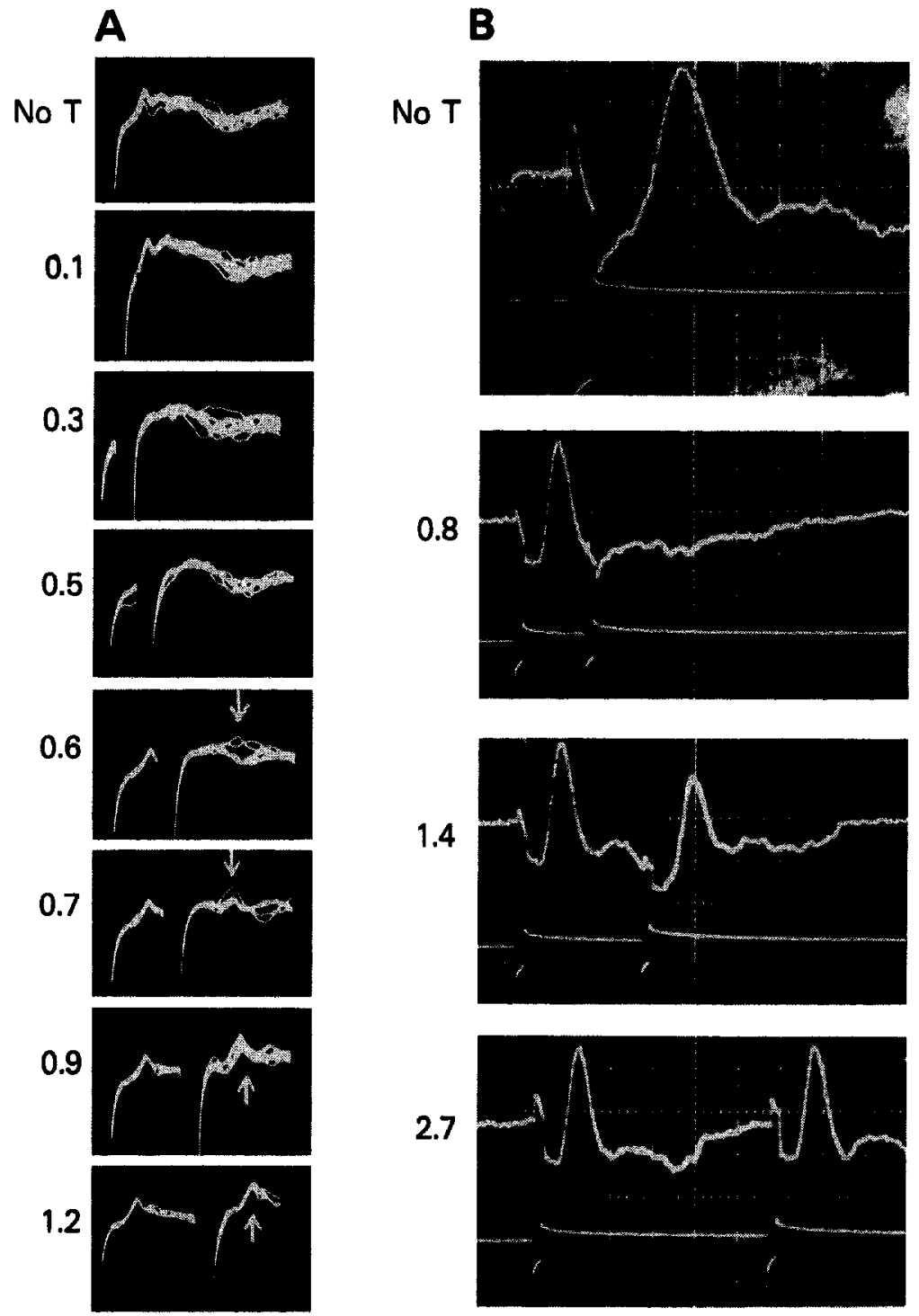

Figure 10. The determination of the refractory periods of electrophysiological responses evoked by stimulation of the medial forebrain bundle. Panel A: Microelectrode recording from a single directly driven unit with a refratory period of $.6 \mathrm{msec}$. (Each photograph shows 5 superimposed sweeps. The C$T$ interval is given on the left. The downwardly disappearing deflections of the trace are artifacts from the stimulating pulses. The action potential following the $\mathrm{C}$ pulse is the small biphasic deflection just as the trace recovers from the artifact. The arrows point to the action potential that follows the $T$ pulse when the C-T interval is .6 msec or greater. [Data collected by E. Rolls, published in Gallistel, 1973.]) Panel B: Compound action potentials produced by a lateral hypothalamic stimulating electrode, as recorded by a ventral tegmental macroelectrode. (The C-T interval is given to the left of each photograph. Vertical sensitivity for bottom three photographs is $200 \mu \mathrm{V} /$ div.; top is double scale. The upper traces are from the recording electrode. The lower traces show the stimulating pulses. Notice that at the shortest C-T interval, .8 msec, the response to the T pulse is absent. [Kiss, Shizgal, \& Rosen, Note 6; see also Deutsch \& Deutsch, 1966.])

component of the MFB (Nauta \& Haymaker, 1969). Electrophysiological experiments show that axons with the inferred re- fractory periods and conduction velocities are in fact fired by rewarding stimulation in the MFB (Deutsch \& Deutsch, 1966; Gal- 
listel, Rolls, \& Greene, 1969; Gallistel, 1973; Rolls, 1971; Rolls, 1975). Figure 10, Panel A shows a refractory period determination on a unit picked up by a microelectrode whose tip was at the dorsal termination of the external medullary (myelinated) lamina of the thalamus, 3-4 mm away from a macroelectrode that delivered rewarding stimulation to the MFB. From the fact that impulses in this unit followed stimulating pulses one for one at an unvarying latency of .35$.45 \mathrm{msec}$, it may be inferred that this unit was directly driven by the rewarding stimulus pulses. From the impulse latency and the distance between the stimulating and recording electrodes, we can calculate a conduction velocity of $7-12 \mathrm{~m} / \mathrm{sec}$. As may be seen in Figure 10, Panel A, the refractory period of this unit was $.6 \mathrm{msec}$ : When the $\mathrm{C}-\mathrm{T}$ interval in the pairs of stimulating pulses was less than $.6 \mathrm{msec}$, there was an action potential following the $\mathrm{C}$ pulses but none following the $T$ pulses. At a $C-T$ interval of $.6 \mathrm{msec}$, there was sometimes an action potential following the $T$ pulse, but more often not. At $\mathrm{C}-\mathrm{T}$ intervals greater than $.6 \mathrm{msec}$, an action potential followed every $T$ pulse. Note, however, that the latency of the action potentials following the $T$ pulse was increased. This is an instance of the retardation of conduction velocity during the period of relative refractoriness, which we have already discussed in connection with Figure 6.

Since the behavioral effects of stimulation are due to the excitation of a bundle of axons, it is of some interest to record the compound action potential in the MFB, which reflects the stimulation-produced activity in the population of axons comprising this tract. Figure 10, Panel B gives photographs from an experiment that determined the refractory periods of the axons contributing to the compound action potential in the MFB. When the T pulse is brought within $.8 \mathrm{msec}$ of the $\mathrm{C}$ pulse, it evokes little if any response. At longer $\mathrm{C}-\mathrm{T}$ intervals, the $\mathrm{T}$ pulse evokes a response comparable to that evoked by the C pulse. Data from several preparations yield estimates for the refractory periods of the contributing axons that range from .6 to $1.6 \mathrm{msec}$.
It must be kept in mind that the contributions of a given axon to the behavioral effect and to the electrophysiological effect may be differently weighted. Furthermore, we have as yet no estimate of what proportion of the electrophysiological response is due to the activity of reward-related axons. These data do establish, however, that the inferences drawn from our behavioral data are physiologically plausible. The ranges of values for MFB refractory periods overlap almost completely.

The electrophysiological and anatomical data pertinent to identifying the substrate for self-stimulation are as yet fragmentary and inadequate. We want to make only two points in regard to these data. First, they establish that axons with the properties we have inferred are in fact fired by rewarding stimulation to the MFB. Second, and more to the theoretical point of our article, anatomical data on myelination and electrophysiological data on refractory periods and conduction velocities are relevant to the question of the identity of the substrate for self-stimulation only because of the behavioral trade-off data. In the absence of the behavioral data, the fact that catecholaminergic axons are unmyelinated with refractory periods of $1.8 \mathrm{msec}$ or greater and conduction velocities of $1.5 \mathrm{~m} / \mathrm{sec}$ or less would have no bearing on the hypothesis that they constitute the first stage fibers in electrical self-stimulation of the MFB. It is our contention that in the absence of behavioral trade-off data it is all but impossible to bring anatomical and electrophysiological data to bear in any telling way on the problem of identifying the substrate for self-stimulation.

Conclusions. Two-electrode paired-pulse data provide the first clear evidence for the widely held belief that the first stage (directly stimulated) tissue underlying MFB self-stimulation consists of axons coursing in the MFB and ventral tegmentum. They also provide the first direct evidence that the self-stimulation sustained by two electrodes several millimeters apart depends on the excitation of one and the same bundle of axons. The estimates of conduction velocity confirm our earlier inference that the bundle consists of small-diameter myelinated axons. 
The two-electrode experiment greatly increases the winnowing power of the behavioral data, by which we mean their power to remove "neural chaff" from consideration. Many different fiber systems are to be found running together at any one site of stimulation. When, however, we consider two stimulation sites several millimeters apart, the number of the systems common to both sites is smaller; hence the winnowing power of these experiments. They lead us to consider only those systems that course through both sites.

The two-electrode experiment provides a powerful approach to the problem of mapping the first-stage neural circuitry subserving stimulation-produced behaviors. The problem of deriving such a neural circuit diagram can be likened to the connect-thedots game found in children's coloring books, where dots refer to effective stimulation sites and lines to the behaviorally relevant axonal pathways. One task is to determine which dot is to be connected with which. The list of sites where behaviors such as self-stimulation or stimulation-bound feeding have been reported is long, and unlike the child's game, the dots are not numbered: One does not know which dots are to be connected to which. Neuroanatomical work suggests possible connections between various dots; but only some of these pathways will turn out to be involved in the behavior under investigation; many of the others will prove to subserve various side-effects of the stimulation. The question of what constitutes a candidate pathway is not simple. In the absence of well established current-distance relationships for central neurons, even the dimensions of the dots are poorly defined.

The approach on which this article is based offers a number of advantages when applied to the mapping problem. First, the excitability and conduction characteristics that are described by the experiments reviewed above are those of the reward-related neurons. If the neuroanatomists have delineated three pathways that connect a particular pair of dots, the quantitative characteristics derived from the behavioral experiments may select the most likely candidate. Second, these experiments describe the behaviorally relevant pathway associated with a particular dot even if nothing whatsoever is known about the trajectory of the stimulated pathway or the identity of its neurotransmitter, that is, even if other neuroscientists have not yet established any pathway between that pair of dots. The resultant characterizations can be used to direct neuroanatomical and neuropharmacological work along lines that are suspected, a priori, of being useful to the student of brain stimulation. Third, paired-pulse experiments and the strength-duration experiments now to be reviewed provide converging, multiple constraints on the kind of pathway that could be the directly stimulated substrate for a stimulation-produced behavior.

Finally, the two-electrode experiment makes it possible to specify, for a given behavior, which pairs of dots are connected by lines. An example of the power of this method is found in Figure 11. These two curves were obtained from the same subject using the same stimulation electrodes and the same currents (Bielajew \& Shizgal, 1980). In one case, the subject was tested for self-stimulation, and a collision effect was obtained; in the second case, the subject was tested for stimulation escape, and no collision effect was observed. It would appear that the circuit diagram for brain stimulation reward should include a line between these two particular dots, whereas the diagram for the aversive effects of stimulation should not.

\section{Current Intensity as a Function of Pulse Duration}

The strength-duration function is a plot of the current required to produce a given level of effect as a function of the duration of the stimulating pulse. The function reflects the current integrating characteristics of the directly excited neural tissue, that is, the extent to which the signal increases as the duration of the exciting current is prolonged. Ranck (1975) has recommended the determination of strength-duration functions as a means of assaying the kind of neural tissue whose excitation underlies a 


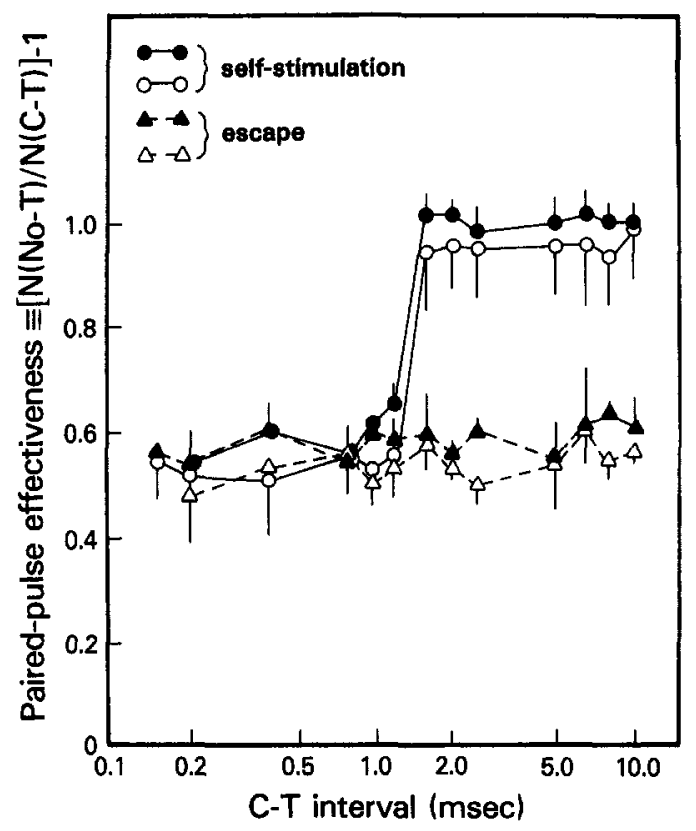

Figure 11. Two-electrode paired-pulse effectiveness for self-stimulation and escape, using the same two electrodes and the same current intensities. (The solid symbols are from the conditions in which the $C$ pulse was on the lateral hypothalamic electrode and the $\mathrm{T}$ on the ventral tegmental electrode. Open symbols are from the conditions in which their order was reversed. The presence of a collision effect [step] in the self-stimulation data and its absence [flat lines] in the escape data imply distinct substrates for these two effects of the stimulation. Vertical bars indicate \pm 1 standard error of the mean. [Adapted from Bielajew \& Shizgal, 1980.])

given behavioral effect of electrical stimulation. He points out that different kinds of tissue-cell bodies, myelinated axons, unmyelinated axons, and so on--have different current integrating characteristics and that different current integrating characteristics will yield different trade-offs between the duration of a stimulating pulse and the strength it must have.

To determine the trade-off between pulse duration and pulse strength, Matthews (1977) had rats run an alley to press a lever that delivered a train of electrical pulses to the MFB. The train had a fixed number of pulses and the interval between pulses was fixed at $30 \mathrm{msec}$. In any one session, the duration of pulses was fixed at a value between .05 and $15.0 \mathrm{msec}$. The rat was run for 11 trials to each of several different cur- rent intensities. This resulted in a plot of the rat's running speed as a function of current intensity, at the pulse duration chosen for that session. The pulse duration was varied from session to session, so that over sessions one obtained a family of running-speed versus current-intensity curves, each at a different pulse duration. From this family of curves, Matthews derived the trade-off between pulse duration and current intensity by plotting the current required to produce half-maximal running speed at each pulse duration. Figure 12, Panel A plots data for both cathodal and anodal pulses against double logarithmic coordinates.

Matthews (1977) and Gallistel (1978) attempted to fit Matthew's cathodal strength-duration data with hyperbolic and exponential functions. The hyperbolic function has the form:

$$
I=r(1+c / d),
$$

and the exponential function has the form:

$$
I=r /[1-\exp (-d / t)],
$$

where $I=$ the required current; $r=$ the rheobase, that is, the current required for indefinitely long pulses; $d=$ pulse duration (width); $c=$ the chronaxie of a hyperbolic function; and $t=$ the time constant of an exponential function. They found that the hyperbolic function fit the data better in every case. However, the fit was not perfect. The data are consistently somewhat flatter than the best-fitting hyperbola. The chronaxies of the best fitting hyperbolas are unusually long, ranging from .7 to $3.0 \mathrm{msec}$, with a mean value of about $1.5 \mathrm{msec}$.

The unusually long chronaxies (and the fact, discussed later, that the priming and reward data are essentially the same) make one wonder whether these data are somehow determined entirely by the type of electrode or some other irrelevant factor and do not reflect anything about the type of tissue being stimulated. To allay these doubts, Matthews also determined cathodal and anodal strength-duration functions for the twitches produced in the somatic musculature by stimulating through these same electrodes at the same or slightly higher current 


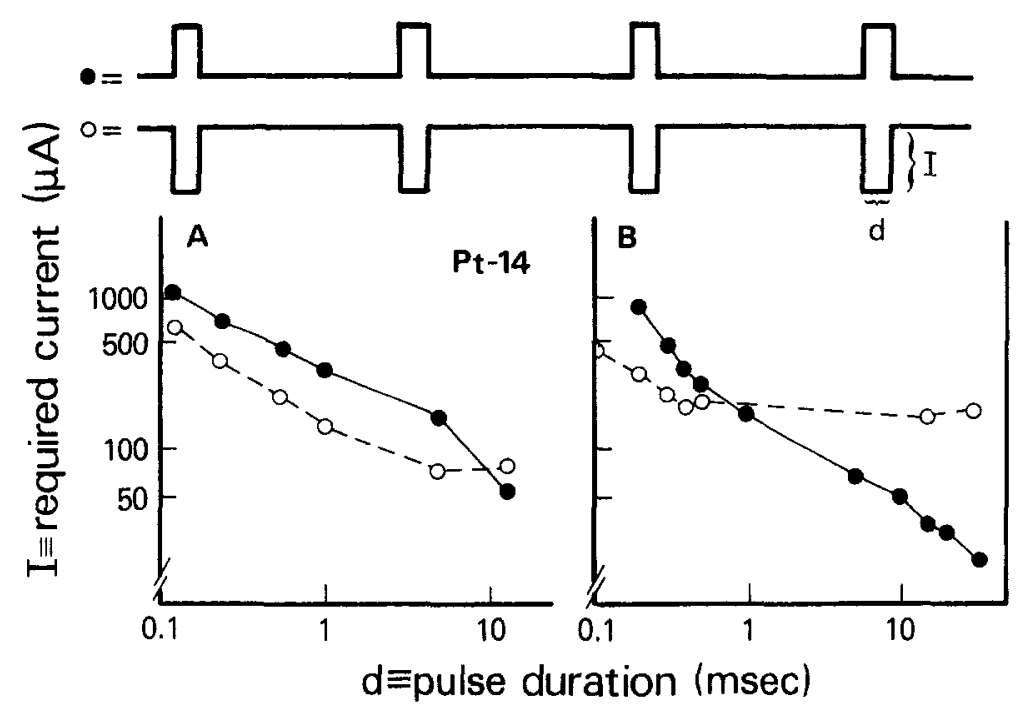

Figure 12. Panel A: Pulse-strength (I) as a function of pulse duration (d) in the production of equivalent rewarding effects, using either cathodal or anodal current. (Both axes logarithmic.) Panel B: Similar data for the motor twitch elicited via the same electrode. (Adapted from Matthews, 1977, Figures 3 \& 8.)

intensities. These twitches presumably reflect the excitation of fibers in the internal capsule, which lies near the MFB. Figure 12, Panel B shows representative data from this experiment. Four things should be noted:

1. Both the cathodal and anodal strengthduration functions for the motor effect differ strikingly from the function for priming and reward. Thus, strength-duration functions are clearly capable of discriminating different kinds of tissue.

2. The cathodal strength-duration functions for the motor effect have short chronaxies. That is, they level off quickly. The chronaxies for the motor effect range from .15 to $.48 \mathrm{msec}$, which is comfortably within the range of chronaxies reported in the neurophysiological literature for single myelinated axons (see Ranck, 1975). One may conclude that the unusually long chronaxies of the strength-duration functions for priming and reward reflect some genuine peculiarity of the tissue mediating these effects.

3. The relation between the anodal and cathodal functions for the motor effect is not the same as the relation between these functions for the priming and rewarding effects. One cannot derive an anodal strength-duration function from the cathodal function by, for example, simply multiplying the cathodal function by a factor of 2 . The characteristics of the underlying tissue that are reflected in an anodal strength-duration curve are different from the characteristics reflected in the cathodal curve.

4. Ranck (1975) points out that the strength-duration function is not determined solely by the membrane characteristics of the behavior-relevant neurons. It depends also on the geometric relation between the relevant neural elements and the pattern of current flow set up by the electrode configuration. For example, Rushton (1930), using the nerve-muscle preparation, showed that the shape of the strength-duration function for the excitation of motor axons was greatly altered when the cathodal and anodal electrodes were positioned in such a way that the current flowed across the axon bundle rather than along it.

\section{The Interpretation of Anodal Functions}

The first puzzle in interpreting anodal strength-duration functions is that it is possible to get a behavioral effect with anodal pulses. Since the entry currents from anodal pulses presumably hyperpolarize the tissue 
immediately surrounding the electrode, one might think that they would fail to fire any action potentials. There are, however, two known ways for an anodal pulse to excite axons. First, the current entering an axon in the vicinity of the electrode must exit from the axon at some more remote area. It is possible for these remote exit currents to fire action potentials. This effect would seem to be more likely in myelinated axons, because the myelin sheath presumably confines the exit currents to small patches of membrane. This mechanism-firing by means of remote exit currents-is the only one likely to work at very short pulse duration. Matthews's data show that at short pulse durations the required anodal current is only about twice as strong as the required cathodal current. This implies that firing by remote exit currents is fairly efficient in the tissue underlying the priming and rewarding effects. This again suggests that the relevant axons are myelinated.

The other mechanism by which anodal pulses may excite action potentials is known as anode-break excitation. During a prolonged hyperpolarization, the sodium gates in the axon membrane become hyperexcitable. This sodium hyperactivation is the converse of the sodium inactivation that occurs during a prolonged depolarization (Hodgkin \& Huxley, 1952). When a prolonged hyperpolarizing pulse abruptly ceases, the rapid return to a normal level of membrane polarization may open the hyperactivated sodium gates enough to cause an action potential. Matthews interpreted the dip in the anodal strength-duration functions at longer pulse durations as indicating the occurrence of anode-break firing.

When anode-break firing occurs, each stimulating pulse may cause one or more action potentials while it is on and another burst of action potentials when it goes off. Matthews was able to confirm this interpretation for the motor-effect data. Since the motor twitches were elicited by single pulses, it was possible to measure the latency between a pulse and the resulting twitch. $\mathrm{He}$ showed that when one excited a twitch with a $15-\mathrm{msec}$ anodal pulse, the latency was 21 msec from pulse offset. When one elicited the same twitch with a cathodal pulse, the latency was $20 \mathrm{msec}$ from pulse onset. These latency measures clearly indicate that when long duration anodal pulses are used a significant fraction of the total firings may be generated at pulse offset.

\section{Electrophysiological Data}

Matthews followed up his behavioral experiments with electrophysiological experiments. He made strength-duration determinations on 27 posterior midbrain neurons directly or indirectly fired by pulses from a monopolar self-stimulation electrode in the MFB (Matthews, 1978). In these experiments, he observed all of the electrophysiological phenomena we have alluded to in discussing the behavioral data. Some neurons could only be fired by cathodal pulses. Others could be fired by anodal pulses as well, but only at pulse onset. Still others could be fired by anodal pulses at both pulse onset and pulse offset. Firing at pulse offset occurred only at longer pulse durations. When it occurred, there was a drop in the required current.

Figure 13 presents a representative sample of these single-unit strength-duration functions. Two of the directly driven units Matthews recorded fired up to three times in response to prolonged cathodal pulses. However, he did not find any units that could integrate cathodal current over more than $5 \mathrm{msec}$. That is, the current required to elicit any number of firings never decreased in response to increases in pulse duration beyond a duration of $5 \mathrm{msec}$. Put another way, the output from all of Matthews' units had ceased by $5 \mathrm{msec}$ after the onset of a pulse.

The last mentioned finding is the most relevant to the present discussion. It allowed Matthews to reject the hypothesis that he had recorded from a representative sample of the axons in the priming or reward cable. The behaviorally determined strength-duration data show a significant drop in required current as the duration of cathodal pulses is increased from $5 \mathrm{msec}$ to $15 \mathrm{msec}$. There is no way to account for this on the basis of Matthews' sample. At the very least, one can say that Matthews' sample cannot be representative. It would have to contain at least one unit that yielded a chronaxie on 


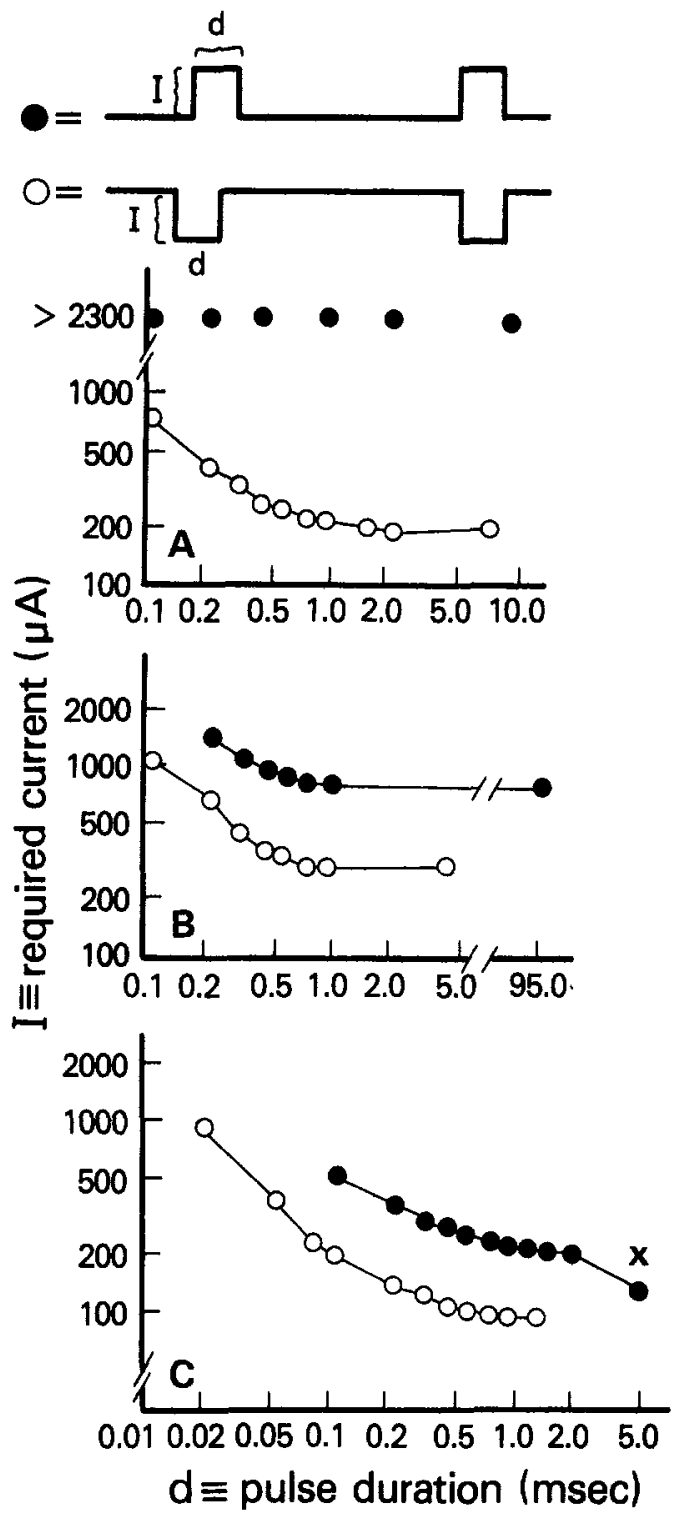

Figure 13. Examples of the strength-duration curves obtained when recording units in the midbrain driven by cathodal or anodal stimulation of the MFB at midhypothalamic levels. (Both axes logarithmic.) Panel A: Data from a single unit that could not be excited by anodal pulses of any duration, even at the maximum available current $(2.3 \mathrm{~mA})$. Panel B: Data from a unit that showed no evidence of anode-break excitation. (The anodal curve is parallel to the cathodal curve.) Panel C: Data from a unit that exhibited both anode-make and anode-break excitation. (The anodal curve is parallel to the cathodal curve at pulse durations up to 2 msec. Between 2 and $5 \mathrm{msec}$, anode-break excitation begins, and there is a drop in required current. The $X$ indicates the threshold for anode-make excitation with a $5 \mathrm{msec}$ pulse. [Adapted from Matthews, 1978, Figure the order of $10 \mathrm{msec}$. In fact, most of the units Matthews recorded yielded chronaxies less that $.5 \mathrm{msec}$, which suggests that these units did not form any part of the cable.

\section{Conclusions}

The data on required current as a function of pulse duration indicate that the axon bundle comprising the first stage of the substrate for self-stimulation integrates cathodal current over unusually long intervals. The output from the bundle (the total number of action potentials) continues to increase for up to $15 \mathrm{msec}$ after pulse onset. The unusually long chronaxie for the cathodal function and the late-occuring dip in the anodal function both imply that these axons accommodate unusually slowly. The dip in the anodal function further implies that they fire on the break of a prolonged anodal pulse. Both the cathodal and anodal functions clearly differentiate the tissue that mediates self-stimulation from the tissue that mediates the motor effects of stimulation. This is particularly interesting because it is likely that the motor-effect axons, like the selfstimulation axons, are myelinated.

As with the paired-pulse data, the strength-duration data give behavioral significance to electrophysiological data whose pertinence to any behavioral phenomenon would otherwise be moot. The fragmentary electrophysiological data so far collected on the strength-duration characteristics of axons driven by rewarding stimulation of the MFB suggest that the requirement that a neural system have the right strength-duration characteristics is a nontrivial constraint on the viable systems. In other words, the winnowing power of these behavioral data is again considerable.

\section{The Integrator}

The integrator in the reward pathway is the postaxonal neural system that summates the signals from the cable over axons (spatial summation) and over time (temporal sum-

3, by permission of ANKHO International, Inc., and the author.]) 
mation). The signal resulting from this summation is the signal seen by the conversion process. The next two experiments reveal properties of this integration.

\section{Current as a Function of the Number of Pulses in a Train of Fixed Duration}

The integrator must sum, over time and space, inputs arriving over the axons that comprise the reward cable. The manner in which this spatiotemporal integration is performed can be described by trading off a stimulation variable that acts over time, such as the number of stimulation pulses, $N$, against a variable that acts over space, such as the current intensity of the stimulating pulses. The data in Figure 14 were obtained in the runway paradigm by determining the currents required to produce a criterial running speed at each of several pulse frequencies (Gallistel, 1978). The train duration was held constant; hence, the number of pulses covaried with pulse frequency. As can be seen from Figure 14, there is a linear relation between required current and the reciprocal of the number of pulses $(1 / N)$. This way of plotting the data was suggested by Shizgal, Howlett, and Corbett (Note 3), who found data similar to those in Figure 14 in the Skinner box paradigm.

The linear relation between required current and the reciprocal of the number of pulses reflects, we believe, two distinct properties of the reward (and priming) pathway - one property of the cable as a whole and one property of the integrator. The cable property is that the number of axons fired is a linear function of current intensity. The integrator property is that the reward-relevant aspect of the integrator's output is determined solely by the number of action potentials delivered to the integrator by the cable, provided that (a) train duration is fixed and (b) the strength of stimulation is constant throughout the train.

Consider, first, the question of the number of reward-relevant axons fired as a function of current intensity. There are good reasons to believe that at short pulse durations, each pulse produces one firing in each of the axons within the region of effective excitation (e.g., Matthews, 1978). The short refractory pe- riods that have been found suggest that axons in the cable should be able to fire one for one at frequencies as high as $400 \mathrm{~Hz}$, the maximum value used in the current-vs-number experiments. If each pulse fires each axon once, then the number of firings seen by the integrator $\left(n_{\mathrm{f}}\right)$ is:

$$
n_{\mathrm{f}}=N \times n_{\mathrm{a}},
$$

where $n_{\mathrm{f}}$ is the total number of firings produced in the cable by a train of pulses; $N$ is the number of stimulation pulses; and $n_{a}$ is the number of axons in the cable excited by each pulse.

Hawkins (see Gallistel, 1976) has suggested that $n_{\mathrm{a}}$, the number of axons excited by a pulse of fixed duration, should be a scalar function of the current intensity. He reached this conclusion by arguing that because flux may be expected to fall off roughly as the inverse square of the distance from the electrode tip, the square of the radius of effective excitation should be directly proportional to current intensity. For straightforward geometric reasons, the number of axons passing within the radius of excitation should be proportional to its square. Hence, $n_{\mathrm{a}}$ should be proportional to $I$. This argument implies that the line relating $I$ to $I / N$ should pass through the origin, in which case there is a precise reciprocity between number of pulses and required current. One notes in Figure 14 that neither for priming nor reward does the line actually pass through the origin, although for reward it comes closer. Hawkins's argument rests on the assumptions that neither the spatial distribution of axons in the cable nor tissue impedance in the region of the electrode tip is radically anisotropic. It has subsequently been argued (Shizgal et al., Note 3) that because of the electrode scar the number of stimulated axons should be a linear rather than scalar function of current:

$$
n_{\mathrm{a}}=k\left(I-I_{0}\right),
$$

where $I_{0}$ is the additive correction factor and $k$ is Hawkins' constant of proportionality. Combining Equations 3 and 4, one obtains the expression

$$
1 / N=k\left(I-I_{0}\right) / n_{\mathrm{f}}
$$




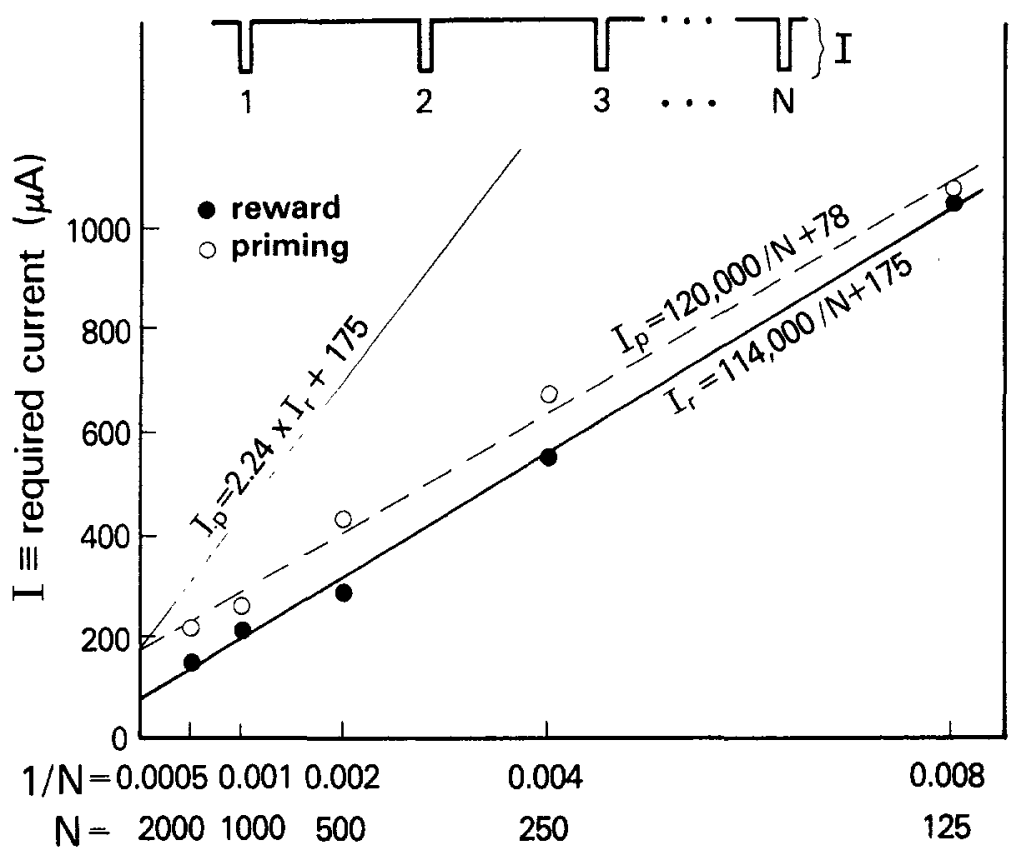

Figure 14. Required current (I) as a function of the reciprocal of the number (N) of pulses in a train. (Solid circles are data for rewarding effect; open circles for priming effect in same rat. Train duration in both experiments was $10 \mathrm{sec}$. Unweighted linear regressions, $I_{z}$ and $I_{p}$, account for $99.7 \%$ and $99.3 \%$ of the variance, respectively. The light line, labeled $I_{p}=2.24 I_{r}+175$, represents the hypothesis that the ratio of the current required for priming to the current required for reward is constant [see section on Priming vs. Reward]. [Reward data published by Gallistel, 1978, in another form; reanalyzed by Shizgal, Howlett, \& Corbett, Note 3.])

or rewriting so as to obtain $I$ on the left:

$$
I=\left[n_{\mathrm{f}} / k\right][1 / N]+I_{0}
$$

Now consider the relation between the number of spikes that the cable delivers to the integrator and the resulting reinforcement-determining output. The number of spikes delivered to the integrator is the variable $n_{\mathrm{f}}$ in Equations 3, 5, and 6. If one supposes that with train duration fixed, the magnitude of the reinforcement depends only on $n_{f}$, then the linear trade-off between $I$ and $1 / N$ is explained: Because the function relating number of pulses and current intensity to performance is monotonic, the function relating the integrator's input to its output must also be monotonic (see Theoretical Foundations). In the trade-off experiment, the reinforcement produced by different combinations of current and number of pulses is held constant. If the reinforcementdetermining output of the integrator depends only on $n_{f}$, ns must also have been held con- stant. Given $n_{\mathrm{f}}$ constant, Equations 5 and 6 express a linear trade-off between $I$ and $1 / N$.

Fixing train duration fixes the duration of the barrage of spikes that the cable delivers to the integrator. To say that integrator output now depends only on $n_{f}$, the number of spikes in the barrage, is to say that the integrator is indifferent to the spatiotemporal distribution of the spikes (see Figure 15 for illustration). The summation processes in the integrator will be indifferent to the spatiotemporal distribution of spikes if either (a) all the spikes in the barrage are given equal weight or (b) the weight given a spike is random with respect to the spike's temporal locus within the barrage and its spatial locus within the population of cable axons. This is equivalent to assuming that the second stage neural network manifests no temporal or spatial facilitation or accommodation. A synapse or neural circuit manifests temporal facilitation if impulses arriving later in a barrage have a greater effect than 

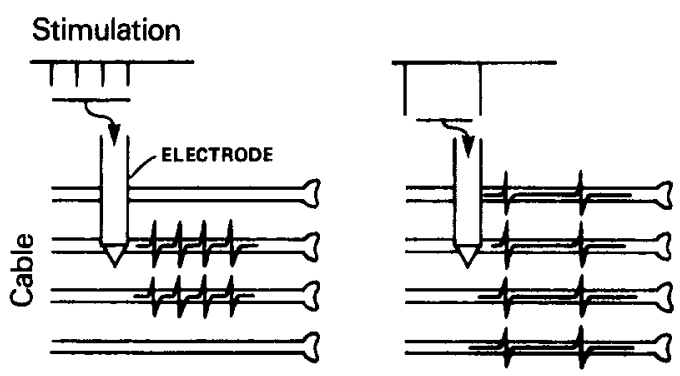

Figure 15. The "counter" model of the integrator assumes that the above two inputs from the cable yield the same output from the integrator, despite the difference in the spatiotemporal distribution of the spikes.

impulses arriving early. Synapses or circuits manifesting temporal accommodation have the opposite characteristic: Impulses arriving late have reduced effectiveness. Analogous nonlinear summation may occur in spatial integration: Impulses arriving simultaneously via several presynaptic pathways may have either a greater or lesser effect than would be expected from simple addition of the individual effects. The occurrence of either sort of nonlinear integration would be inconsistent with our assumption that the integrator weights incoming spikes randomly with respect to their temporal and spatial position.

Conclusions. To account for the linear relation between $I$ and $1 / N$, we have made three closely intertwined assumptions: (a) Each .1-msec stimulating pulse fires each reward-relevant axon at most once. (b) The number of reward-relevant axons fired is a linear function of current intensity. (c)When one fixes the duration of a barrage of impulses seen by the integrator, the integrator behaves as a monotonic "counter," that is, its output is determined solely by the number of impulses in the barrage. The strength of the argument derives from the close intertwining of these assumptions. If one alters any one of the assumptions, one must alter at least one of the others as well, in order to explain the trade-off between number and current. Although one can think of several plausible alernatives to any one of these assumptions, the necessary alterations in the other two assumptions seem ad hoc and implausible.

In short, to account for the linear rela- tionship between $I / N$ and $I$, nonlinearities in the cable and/or integrator must be introduced in compensatory pairs, trios, and so on. There does not seem to be any reason to expect precisely compensatory nonlinearities. The linear trade-off is most readily explained by assuming that the reward depends only on the duration of the barrage and the number of spikes in it, and further assuming that the number of axons fired (hence number of spikes) increases linearly with current.

\section{Required Charge and Charge/Sec as Functions of Train Duration}

Neural networks with one or more synaptic relays commonly behave as leaky integrators. The temporal summation they perform depends on the effects of later synaptic inputs summating with a decaying residue from earlier inputs. The greater the interval between inputs, the less the residue, hence the less the summation between the effects of the temporally dispersed inputs.

One often studied manifestation of temporal integration is the relation between the duration of an input and the required strength of that input. When the input is short, it must be strong to create the required effect within the short time allotted. When the input is long, it need not be so strong. Longer inputs are, however, less efficient. The longer interval between the initial and final parts gives more time for initial effects to decay before final effects are felt. Hence there is less summation between the effects of initial and final parts. When the duration is so long that the effects of the initial part have decayed completely by the time the final parts are delivered, one has reached the limits of temporal integration. The required strength of the input no longer decreases as the duration increases. The duration at which the strength-duration function approaches its minimum (the rheobase) indicates the limit of temporal integration.

Another, less common way of examining strength-duration data is to plot the total input required (the integral of strength over time) as a function of input duration. Because long inputs are less efficient, the required total increases as input duration increases. The nature of the data obtained in 
the following experiment make this the best way of displaying the data.

The experiment (Gallistel, 1978) used the runway paradigm. Pulse frequency was set at $100 \mathrm{~Hz}$ and pulse width at $.1 \mathrm{msec}$. Train duration varied from session to session. At each train duration, Gallistel determined the current required for half maximal running, from which he calculated the required charge, $Q$. As shown in Figure 16, the required charge is a linearly increasing function of train duration: that is,

$$
Q=R D+Q_{0}
$$

where $D$ is train duration, $R$ is the slope of the line, and $Q_{0}$ is the zero intercept (the charge required as train duration goes to zero).

If required charge is a linear function of train duration, then dividing Equation 7 through by $D$, we see that the required strength of a train is a hyperbolic function of train duration:

$$
\begin{aligned}
\dot{Q} & =Q / D=R+Q_{0} / D \\
& =R\left[1+\left(Q_{0} / R\right) / D\right]=R(1+C / D),
\end{aligned}
$$

where $\dot{Q}$ is the strength of the train (in microcoulombs $/ \mathrm{sec}$ ), $C=Q_{0} / R$ is the chronaxie of the hyperbola, and $R$ becomes the rheobase of the strength-duration curvethe minimum strength required as $D$ becomes indefinitely long. Figure 17 gives strength-duration data for two rats, plotted on $\log -\log$ coordinates.

Discussion. The chronaxie, $C$, of the hyperbola is an index of the capacity for temporal integration. Empirically, $C$ represents the duration at which the required strength is twice the rheobase. The longer $C$ is, the longer the interval over which there is any appreciable temporal integration. Gallistel found that the chronaxie was about half a second ( $M=.45 \mathrm{sec}, S D=.11$, across 12 rats). This means that there is very little temporal integration beyond about $2 \mathrm{sec}$. Similar estimates of the limits of temporal integration in the reward pathway may be derived from other studies employing very different methods (Deutsch, Roll, \& Wetter, 1976; Milner, 1978; Shizgal \& Matthews, 1977-see Gallistel, 1978, for discussion).
The concept of the strength $(\dot{Q})$ of a train of pulses needs some comment. The strength is not the current intensity. It is the charge delivered per second, which is the product of the charge per pulse, $q$, and the pulse frequency. Stimulation at $50 \mathrm{~Hz}$ and 200 $\mu \mathrm{A}$ has the same strength as stimulation at $10 \mathrm{~Hz}$ and $1,000 \mu \mathrm{A}$. The study by Gallistel (1978) on number-current trade-offs (reviewed above) showed that in most cases there is an approximate reciprocity between the number of pulses and the current intensity (that is, the line relating $I$ to $1 / N$ passes near the origin; see Figure 14). To the extent that there is reciprocity, the required strength of a train is independent of pulse frequency.

\section{Is the Integrator a Linear System?}

The linear relation between required current and the reciprocal of the number of pulses suggests that the integrator is a linear system. A linear system is a system whose output to two inputs delivered concurrently is the sum of its outputs to the inputs delivered alone. If the integrator is a linear system, its behavior is completely characterized by its impulse response, that is, its response to an extremely brief train. The question arises whether it would be possible to determine the impulse response from strengthduration data.

In order to answer this question, one must make an assumption about what aspect of the integrator's output the conversion process looks at. One must specify, in other words, the reinforcement-relevant aspect of the integrator's output. Norman and Gallistel (1978) assumed that the conversion process looks at the peak of the integrator's output, as the homunculus in Figure 2 is doing. In other words, they assumed that the engram represents the peak of the transient signal that produced it, much as the ring left on a bathtub represents the peak level of the transient bath water. They showed that under this assumption, the strength-duration function for rectangular inputs does not determine an impulse response for the integrator. (Rectangular inputs are inputs of constant strength, such as Gallistel, 1978, used.) Norman and Gallistel showed, however, that it is possible, at least in principle, to deter- 


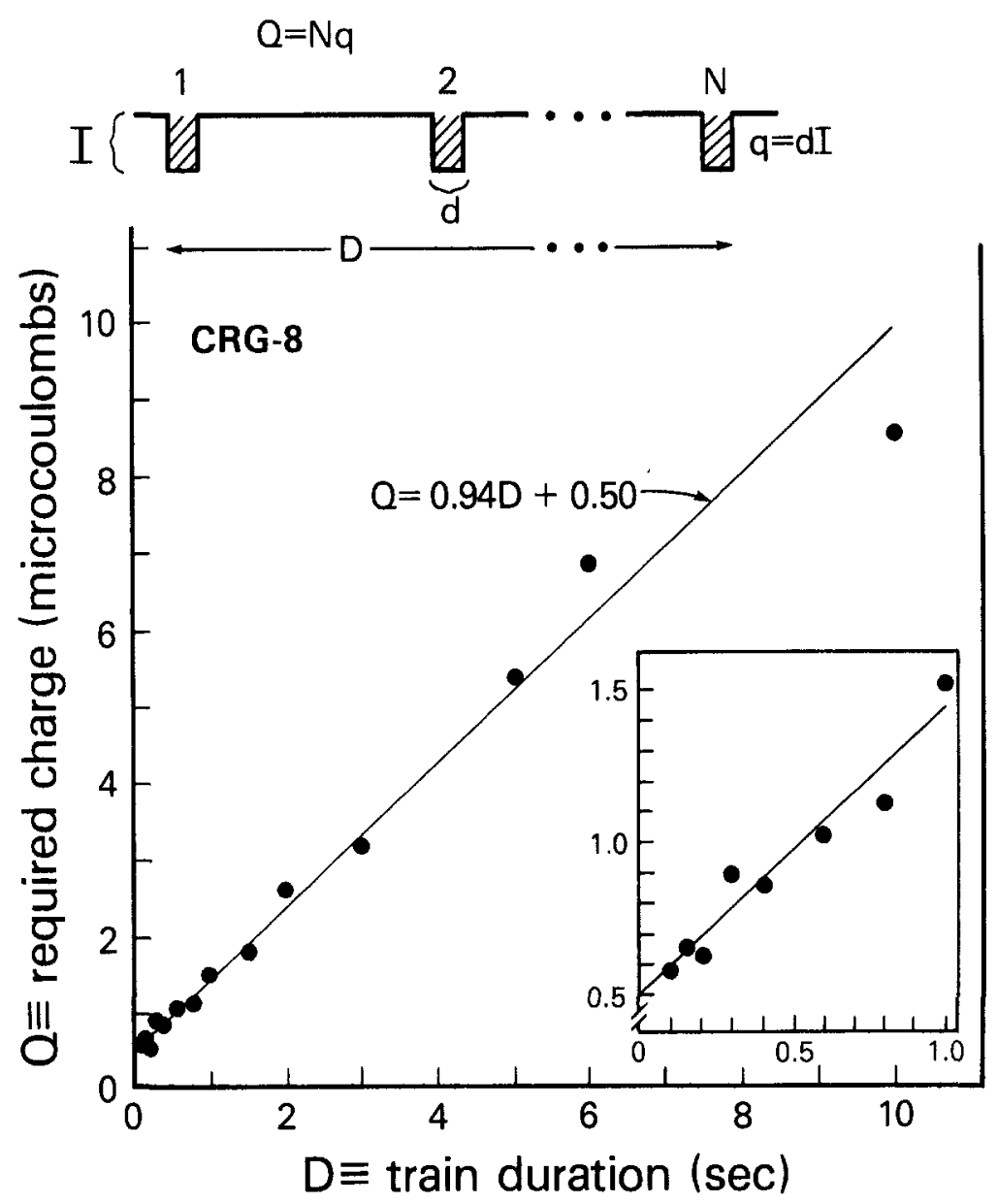

Figure 16. Required charge (Q) as a function of train duration (D). The required charge is $\mathrm{Nq}$, where $\mathrm{q}$ is the charge per pulse and $\mathrm{N}$ is the number of pulses. (The center line, labeled $\mathrm{Q}=.94 \mathrm{D}+.50$, represents the best fitting [weighted] linear regression. [Adapted from Gallistel, 1978, Figure 3.])

mine the impulse response using exponentially decaying inputs.

\section{Does the Integrator Leak?}

We would like to have a model of the integrator that accounts in some straightforward way for the startling linearity of the charge-duration function. We have assumed that the integrator is a leaky one, but unpublished attempts by one of us (Gallistel) to model the integration have not turned up a simple model that would yield this result. One way to obtain this result, however, is to abandon the assumption of leaky integration and assume instead perfect integration above some threshold. The assumption that the integration process sums, without loss over time, the effects of all impulses in the cable that exceed some threshold number per second yields a linear charge-duration function. The steady increase in charge as duration increases is due to the steady wastage of the below-threshold signal. This summator, like all physically realizable summators, would have to have a limit on its capacity. The maximum possible reward would be the one that filled the summator to capacity. Tests may readily be devised that will distinguish between this model and any sort of leaky integrator. 


\section{The Priming Pathway}

So far, we have only discussed the quantitative characteristics of the reward pathway in the minimal model. In this section we review data on the priming pathway, focusing particularly on the question of whether it may be distinguished from the reward pathway on the basis of its quantitative characteristics.

The first attempts to use behavioral experiments to determine quantitative properties of the neural substrate for self-stimulation seemed to find differences in the refractory periods of the neurons mediating the priming and rewarding effects (Deutsch, 1964; Gallistel, Rolls, \& Greene, 1969). These first experiments did not, however, determine trade-off or "equivalent-stimuli" functions. Rather, they determined "inputoutput" functions (Gallistel, 1975). They plotted performance (rate of pressing or running speed) as a function of the $\mathrm{C}-\mathrm{T}$ interval. Yeomans (1975) pointed out that the conclusions drawn from these data were therefore suspect (see also Gallistel, 1975, Figure 8).

Subsequent experiments have justified
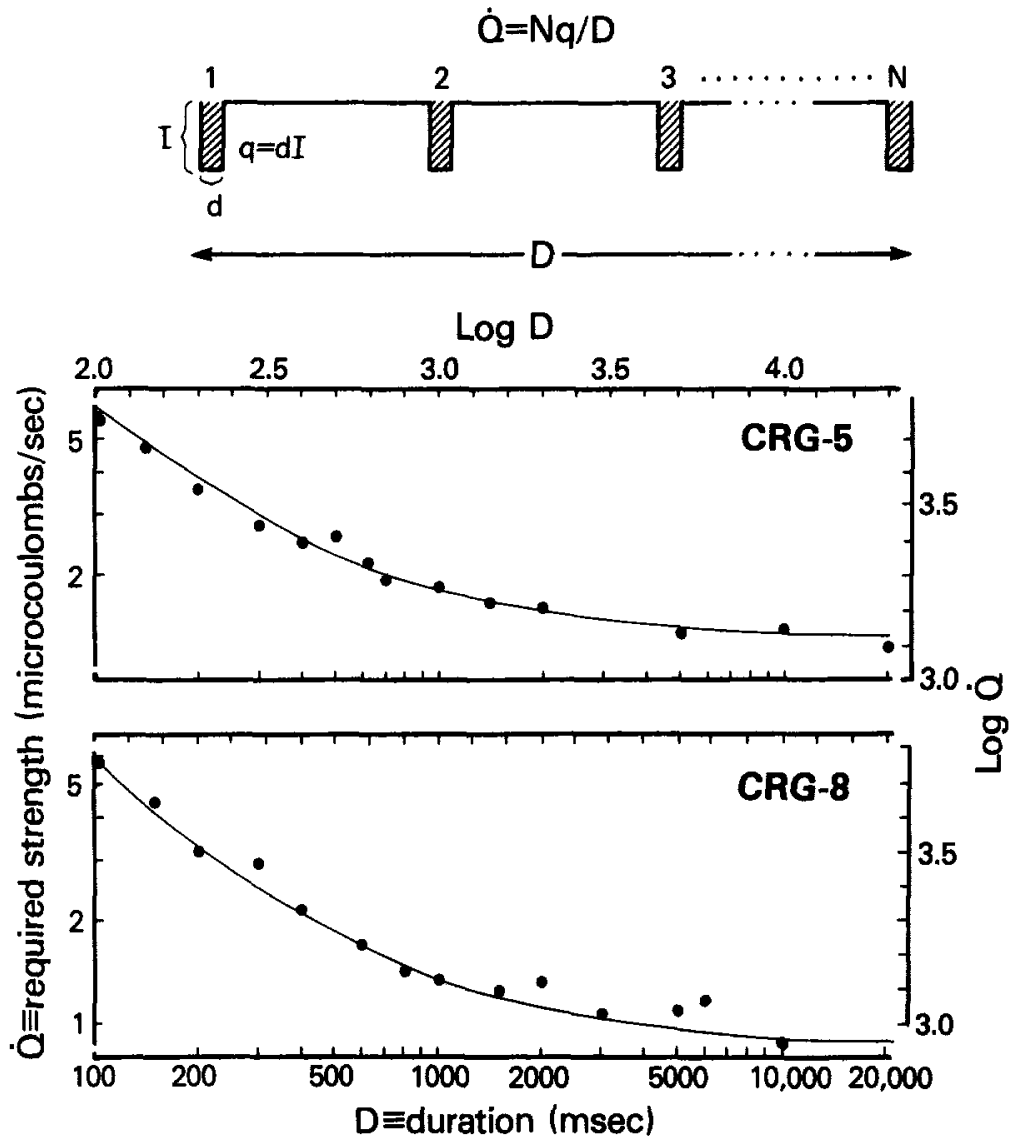

Figure 17. Required train strength ( $\dot{Q}=$ charge per second) as a function of train duration. (The data in the lower panel are the same data as in Figure 16, replotted in strength-duration form. The solid line is calculated from a hyperbolic strength-duration function [8], using a representative value for the chronaxie $[.5 \mathrm{sec}]$. Slightly better fits are obtained using differing values of chronaxie for each data set- $.35 \mathrm{sec}$ for CRG-5, and .53 sec for CRG-8. [Data originally published by Gallistel, 1978, in chargeduration form.l) 
these suspicions. The data now to be reviewed show that the axons mediating the priming and rewarding effects have indistinguishable refractory period and current integrating characteristics. From this one may conclude either that the same axons-hence the same initial signal-mediate both effects or that the two effects derive from the excitation of distinct populations of axons with the same refractory period and current integrating characteristics.

\section{Refractory Periods for Priming and Reward}

Gallistel (unpublished data) determined current versus $\mathrm{C}-\mathrm{T}$ interval functions for priming and reward, using the automated runway paradigm and a rat with an electrode in the posterior lateral hypothalamus. To determine the function for reward, the priming stimulation, which the rat received just prior to each trial, was held constant at 10 trains, 64 pulses/train, .1-msec pulse duration, $100 \mathrm{~Hz}$, and $400 \mu \mathrm{A}$. The duration of the train of paired pulses that the rat received as a reward for running was $10 \mathrm{sec}$, and the interval between pairs of pulses was $40 \mathrm{msec}$. The current required to sustain a half maximal running speed was determined 4 times at each of $5 \mathrm{C}-\mathrm{T}$ intervals and in the no- $T$ condition.

To determine the function for priming, the reward for each run was set at 1 train, 64 pulses, $100 \mathrm{~Hz}$, and $400 \mu \mathrm{A}$. The $\mathrm{C}-\mathrm{T}$ intervals and other parameters of the single train of priming stimulation were the same as for the determination of the function for reward. The performance criterion for the determination of required current was a running speed half way between the maximal running speed and the no-priming running speed. There were 5 determinations of required current under each of the 6 conditions (baseline condition and $5 \mathrm{C}-\mathrm{T}$ interval conditions).

In order to compare the refractory period data for priming and reward, Gallistel computed paired-pulse effectiveness (Formula 2). For statistical reasons, the computation was carried out in the logarithmic domain.
That is, he computed

$$
\overline{\log I(\text { No-T })}-\overline{\log I(\mathrm{C}-\mathrm{T})}
$$

and then took the antilog. When the computation is carried out in the logarithmic domain, the variance of the statistic is the sum of the component variances. This variance was used to compute $95 \%$ confidence intervals and perform $t$ tests on the primingreward comparisons.

There were no significant differences in paired pulse effectiveness for priming versus reward at any C-T interval except the longest (see Figure 18). At the longest $\mathrm{C}-\mathrm{T}$ interval $(20 \mathrm{msec})$, the train consisted of evenly spaced pulses at twice the baseline frequency; the pairing existed only in the mind of the experimenter. The difference between the priming and reward data at a C-T interval of $20 \mathrm{msec}$ does not indicate a difference in the recovery from refractoriness. Rather, it indicates a significant difference in the ratios between the slopes and intercepts of the functions relating the number of relevant axons fired to current intensity (compare Figure 14; data from same animal). Formula 2, which was used to compute paired-pulse effectiveness, implicitly assumes that the effectiveness of a pulse is a scalar function of current intensity. In fact, as explained above, the effectiveness of a pulse is a linear rather than scalar function of current. It is easy to devise a formula for paired-pulse effectiveness that takes account of the linear nature of the scale, but there is no easy way to compute a variance for the resulting statistic. Hence, this formula cannot be used to make null-hypothesis testing comparisons between priming and reward data.

This experiment has only been done on one rat, and current rather than the number of pulse pairs was used to offset the effects of varying $\mathrm{C}-\mathrm{T}$ interval. The results, therefore, are in no way conclusive. They do, however, cast doubt on the conclusions drawn from earlier experiments using input-output paradigms, which seemed to find differences in the refractory periods for priming and reward (Deutsch, 1964; Gallistel et al., 1969). 


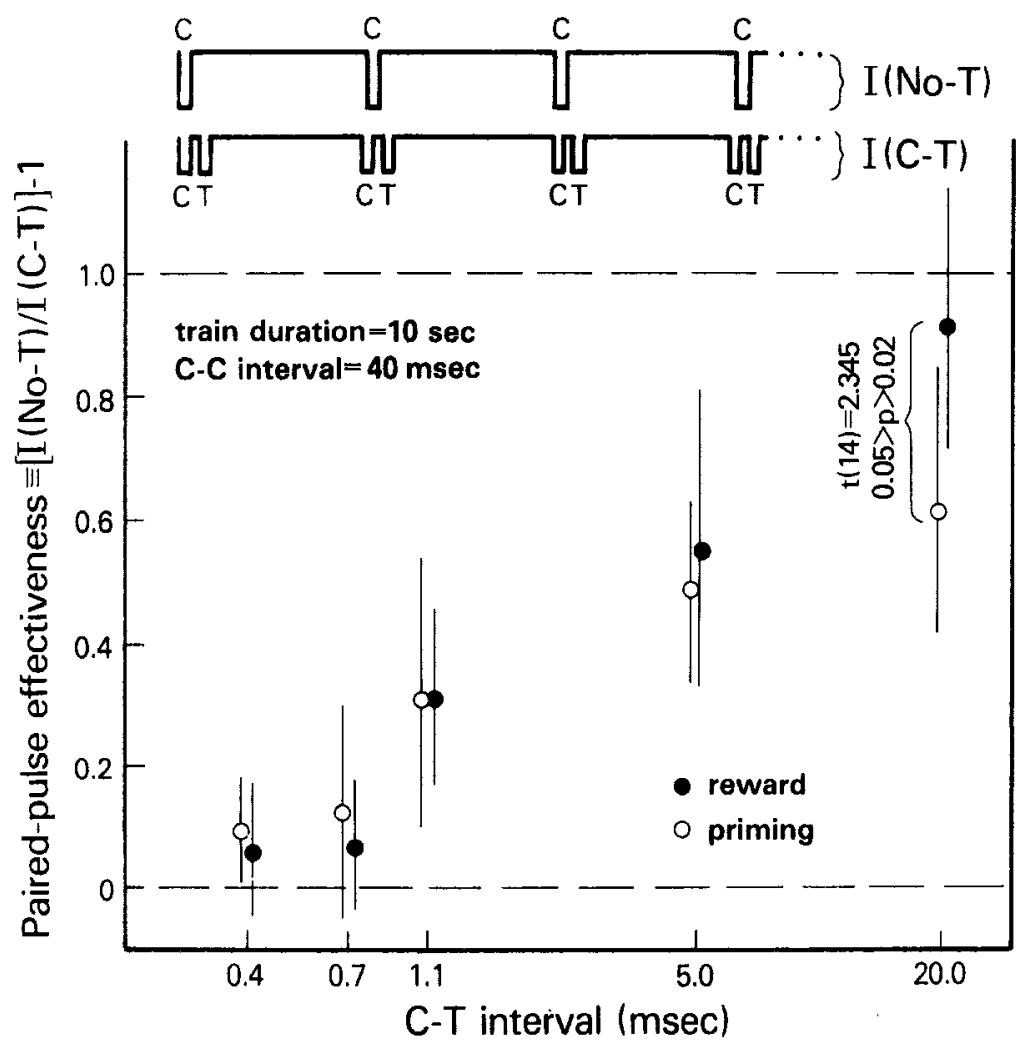

Figure 18. Paired-pulse effectiveness as a function of $\mathrm{C}-\mathrm{T}$ interval for both the priming and rewarding effect of stimulation through an electrode in the posterior lateral hypothalamus. (Each point is based on the ratio of two means. The computation of these ratios and their variance was carried out in the logarithmic domain. Vertical bars give $95 \%$ confidence intervals.)

\section{Pulse-Intensity Versus Pulse-Duration Functions for Priming and Reward}

Matthews (1977) determined cathodal and anodal pulse-strength versus pulse-duration functions for the priming effect as well as for the rewarding effect. To measure the priming effect, he used the runway paradigm. He set the stimulation received as a reward for running an alley at a constant value and varied the pulse width and pulse intensity in the priming stimulation, which the rat received just prior to each trial.

The strength-duration functions for priming and reward were determined in the same rats. Figure 19 shows a rat by rat comparison of the cathodal functions for priming and reward. The differences between the priming and reward data are negligible. They no- where exceed the bounds of experimental error. The anodal functions are similarly congruent.

The failure to find differences in the strength-duration functions for priming and reward is not due to any gross insensitivity of this measure to genuine differences in current integrating characteristics. Matthews also determined strength-duration functions for the motor effects of the same stimulation in the same rats. One could be fairly certain that these motor effects were mediated by a population of axons distinct from the population mediating the priming and reward effects. Both the anodal and cathodal strength-duration functions for the motor effect differed markedly from the corresponding functions for priming and reward (Figure 12). 


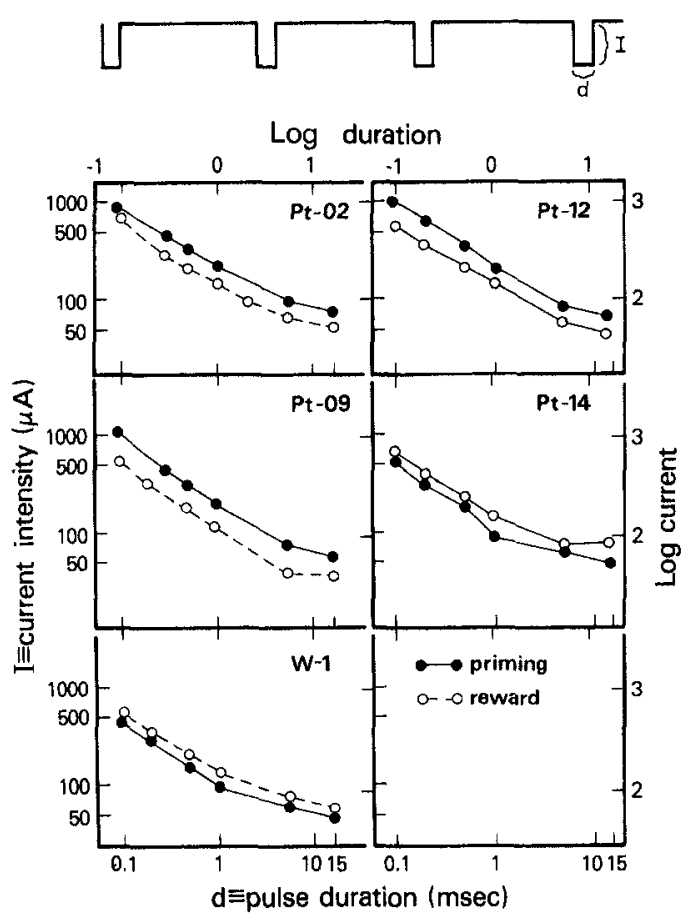

Figure 19. A comparison of required current (I) as a function of pulse duration (d) for priming and reward in five rats. (Adapted from Matthews, 1977.)

\section{Conclusions}

The trade-off data on the refractory period and current integrating properties of the axons whose direct excitation produces priming and reward do not reveal any differences in these properties. The axons in the priming cable appear to have the same properties as the axons in the reward cable. The parsimonious interpretation is that the two cables are one and the same. The data do not, however, exclude the possibility that there are two distinct cables composed of axons with indistinguishable refractory period and current integrating properties.

\section{Current Versus Number of Pulses: Priming-Reward Comparison}

The data in Figure 14 on the relation between the number of pulses in a train and the required current were obtained from the same animal that generated the refractory period data in Figure 18. Referring back to Figure 14 , one notes that for the priming effect, as for the rewarding effect, the required current is a linear function of the reciprocal of the number of pulses in the train. From this relation, for reasons given above in connection with reward, we infer that the number of priming-relevant axons fired by a pulse is a linear function of current.

At first glance, Figure 14 seems to provide further evidence that the priming and reward cables are if not identical, then extremely similar. In fact, however, Figure 14 reveals a significant difference between them: The regression of current, $I$, on $1 / N$ for priming closely parallels the regression of $I$ on $1 / N$ for reward. One is tempted to assume that the only reason the two regression lines are not superimposed is that the criterion used in deriving the priming trade-off must have required more total firings than the criterion used in deriving the reward trade-off. But this cannot be the explanation. The curves relating running speed to current at various fixed values of $N$ are parallel when plotted against a logarithmic current axis. Trade-off functions derived from such a family of curves using different performance criteria differ by a constant ratio (Edmonds, Stellar, \& Gallistel, 1974). What Figure 14 reveals instead is a fixed difference between the currents required for priming and reward. The fixed ratio hypothesis is represented by the light line, labeled $I_{p}=2.24$ $I_{\mathrm{r}}+175$, in Figure 14. It is clearly falsified by the data. The statistically significant difference at a $\mathrm{C}-\mathrm{T}$ interval of $20 \mathrm{msec}$ in Figure 18 may be shown to be a consequence of this failure of the fixed ratio hypothesis.

We have only recently appreciated the character of this difference in the currentnumber trade-off for priming and reward. In order to assess its theoretical significance, we would need a verified theoretical interpretation for the value of the intercept in the function relating $I$ to $1 / N$. At the moment we have none.

\section{Charge or Charge/Sec Versus Train Duration: Priming-Reward Comparison}

Liran (unpublished experiments), used the runway paradigm to determine required charge as a function of train duration for both the priming and the rewarding effect 
in a group of rats with posterior MFB placements. For priming, as for reward, required charge is a linearly increasing function of the train duration, or, equivalently, required train strength decreases hyperbolically to a minimum as train duration increases. Furthermore, the chronaxies for priming and reward are comparable (see Table 1).

In short, when temporal integrating characteristics in the priming and reward systems are assessed by the trade-off between the duration of a single train and its strength, the integration has closely similar properties in the two systems. This frankly puzzles us. Gallistel (1969) reports data showing clearly that when a rat is primed with several trains of stimulation with half-second intervals between trains, temporal integration in the priming effect extends over 10-20 sec. Shizgal (1975) found confirmatory data in another task. Shizgal and Matthews's (1977) data show that, by contrast, there is no integration in the reward system over trains spaced $1 \mathrm{sec}$ apart. It would appear that the priming system is more effectively driven by interrupted stimulation than by continuous stimulation. This conclusion would seem to require a nonlinear model of integration in the priming system - a model in which adaptation to continuous stimulation occurs at some intermediate stage.

\section{Summary of the Findings}

Both the priming effect and the reinforcing effect of rewarding electrical stimulation of the MFB have been investigated by trade-

Table 1

Data on Reward and Priming Chronaxies

\begin{tabular}{lcc}
\hline $\begin{array}{c}\text { Animal- } \\
\text { electrode }\end{array}$ & $\begin{array}{c}\text { Reward } \\
\text { chronaxi } \\
(\mathrm{sec})\end{array}$ & $\begin{array}{c}\text { Priming } \\
\text { chronaxie } \\
(\mathrm{sec})\end{array}$ \\
\hline T11-E & .86 & .17 \\
T12-E & .54 & .89 \\
T12-H & .78 & .28 \\
T3-H & .36 & .15 \\
L27-E & .53 & .49 \\
LB1-E & .47 & .30 \\
Mean $^{\mathrm{a}}$ & .59 & .38 \\
\hline
\end{tabular}

${ }^{a}$ Paired comparisons test for difference between the means: $t(5)=1.42, p>.2$, two-tailed. off experiments in order to place quantitative constraints on the neural substrate. The first kind of experiment trades the number of pairs of pulses against the within pair $(\mathrm{C}-$ $\mathrm{T})$ interval, with both pulses being delivered via the same electrode. The data show two phases: an initial phase of declining pulsepair effectiveness as the $\mathrm{C}-\mathrm{T}$ interval increases from 0 to $.5 \mathrm{msec}$, followed by a phase of recovering effectiveness as the interval increases beyond $.5 \mathrm{msec}$. We attribute the first phase to local potential summation and the second to the recovery from refractoriness. The second kind of experiment is the same as the first, except the pulses in a pair are delivered via different electrodes several millimeters apart along the MFB. The data show only one phasea recovery of effectiveness, which occurs later than the recovery seen in the one-electrode data. From the difference in the onset of recovery in the two sets of data and the distance between the two electrodes, we calculate conduction velocities of $2-8 \mathrm{~m} / \mathrm{sec}$. The third kind of experiment trades current intensity against pulse duration for both cathodal and anodal pulses. The cathodal strength-duration functions have unusually long chronaxies $(1.5 \mathrm{msec})$. The anodal functions lie about $.3 \mathrm{log}$ units above the cathodal functions for pulse durations up to about $5 \mathrm{msec}$, which is to say that the required anodal current is twice the required cathodal current. Beyond $5 \mathrm{msec}$, the anodal curve dips down to, or even below, the cathodal curve. The data from the first three experiments suggest that the first-stage neural tissue consists of long, thin, slowly accommodating, myelinated axons.

The fourth kind of experiment trades the current intensity against the number of pulses in trains of fixed duration. The required current is a linear function of the reciprocal of the number of pulses, with an intercept that is usually small but non-zero. We interpret this to mean that the number of first-stage axons fired is a linear function of current intensity and that the integrator is indifferent to the spatiotemporal distribution of action potentials in barrages of constant strength. The fifth kind of experiment trades the total charge (or the charge delivered per second, depending on how one 
chooses to look at it) against the train duration. The required charge is a linear function of train duration, which means that required charge per second is a hyperbolic function of train duration. The chronaxie of the hyperbola is about $.5 \mathrm{sec}$, which means that the curve is very nearly flat by $2 \mathrm{sec}$. We interpret this to mean that the limit of temporal integration is about 2 sec.

In four of the experiments, the data for priming and reward are very similar. The two-electrode paired-pulse experiment for the priming effect has yet to be done, but it does not appear that these five kinds of experiments are likely to reveal any sizable differences in the quantitative characteristics of the substrates for priming and reward. The experiments do, however, reveal differences between the substrate for MFB selfstimulation and the substrates for the aversive and motor effects of stimulating at the same sites. They also reveal differences between the substrate for MFB self-stimulation and the substrates for self-stimulation at sites in the periaqueductal grey and in the medial frontal cortex.

\section{Pharmacological Properties}

Attempts to determine the pharmacology of the reward pathway in self-stimulation may be viewed as an extension of the reductionist methodology here expounded. Experiments in behavioral pharmacology can add pharmacological properties to the list of properties a neural system must manifest in order to be regarded as the reward system. The behavioral experiments must, however, be designed in such a way that one can confidently attribute the observed effects to an action of the drug on the reward system itself rather than on some other system relevant to the performance of the rewarded behavior. As Wise (1978), Rolls, Kelly, and Shaw, (1974), and Fibiger, Carter, and Phillips (1976) have all pointed out, the experimental paradigms most commonly employed to assay the effects of drugs on self-stimulation do not permit any such attribution.

Two paradigms do, however, appear to permit such an attribution; and both yield data showing that the dopamine blocker pimozide reduces or abolishes the reinforcing effect of stimulation. The first such paradigm is the extinction paradigm (Fouriezos \& Wise, 1976; Fouriezos, Hansson, \& Wise, 1978; Franklin \& McCoy, 1979). The extinction-like change in performance seen under pimozide may not be entirely due to the blocking of reinforcement (Ettenberg, Cinsavich, \& White, 1979; Phillips \& Fibiger, 1979). However, the inclusion of some additional controls has shown that whatever else may be going on, the extinction observed under pimozide is genuine (Gallistel, Boytim, Gomita, \& Klebanoff, Note 4). The conclusion that pimozide blocks reinforcement has been confirmed in a second paradigm, based on shifts in the reward summation function. This paradigm was developed by Edmonds and Gallistel (1974, 1977) and used by Franklin (1978) to evaluate pimozide. On the basis of these experiments, it seems that a further property of the reward system is that pimozide blocks the conversion of the reward signal into an engram.

Our data seem to rule out the assumption that catecholamine neurons constitute the first stage of the reward pathway, leaving open the following possibilities for explaining the action of pimozide: Some or all of the neurons in the second stage may utilize dopamine as a transmitter, in which case pimozide would block the output from the second stage; or it may be that none of the neurons in either stage is dopaminergic. Pimozide may not act upon the reward pathway as such (i.e., upon Stages 1 or 2 in Figure 2); rather, it may block the conversion process-either directly or by blocking some tonic input without which the conversion process cannot proceed. It is worth noting in this connection that pimozide does not block the priming effect of rewarding stimulation, only its reinforcing effect (Gallistel et al., Note 4; Wasserman \& Gallistel, Note 5).

\section{The Theoretical Foundations}

Statements about the refractory periods, conduction velocities, and current integrating characteristics of axons usually derive from electrophysiological experiments. The properties in our portrait of the substrate for self-stimulation were inferred from experiments in which the dependent variable was 
the running speed or bar pressing rate of the rat. Since an unspecifiably large number of distinct neural systems and distinct neurophysiological processes contribute to any behavioral performance, one is inclined to assume that precise knowledge about the physiological processes early in the underlying chain of causes and effects cannot be known from behavioral observations. This is false. Two sorts of behavioral experimentsdifference in reaction time experiments and trade-off experiment-have been used for more than 100 years to reveal quantitative properties of the neurophysiological events that mediate behavior. In this section we explain why trade-off data have this power and why the derivation by behavioral methods of quantitative aspects of the underlying neurophysiology plays an indispensable role in linking neurophysiology to behavior.

Trade-off experiments have played an important role in the establishment of quantitative constraints on neurobehavioral systems. All the basic phenomena of axonal conduction were established by trade-off experiments that used the twitch of the innervated muscle as the dependent variable. The all-or-none principle (Adrian, 1912, 1914), the poststimulation excitability cycle (Adrian, 1916; Adrian \& Lucas, 1912), the strength-duration characteristics of axons, and the resulting models of excitation and accommodation (Hill, 1936; Hoorweg, 1892; Lapicque, 1907) were all established by behavioral methods. The experiments that established the quantitative characteristics of local and conducted excitation in axons relied on a behavioral consequence of conducted excitation that, like all behavioral phenomena, was a remote consequence. Several processes that were then poorly understood intervene between axonal excitation and the muscle twitch. Yet the trade-off experiments were able to see through these intervening processes to reveal the characteristics of the excitation process in axons.

It may seem that the success of work on the nerve-muscle preparation is not a severe test of the power of the trade-off method. The running speed of the rat is a much more remote consequence of the excitation of axons in the MFB, and processes as obscure as engram formation are known to intervene.
A more apropriate example of the power of the trade-off method is the determination of the action spectrum of rhodopsin on the basis of human verbal responses. The in situ action spectrum of rhodopsin was first determined by showing flashes of dim light at various wavelengths to a dark-adapted human observer and adjusting the intensity until the observer's frequency of seeing (frequency of yes responses) met some criterion (e.g., $50 \%$ ). The resulting function is the human scotopic spectral sensitivity function. This function, which derives from human verbal reports, is superimposable on the function a photochemist obtains when measuring the amount of light required at various wavelengths in order to isomerize a given fraction of the rhodopsin in a test tube (when correction for ocular absorption is made).

It is increasingly appreciated that in order to link modern electrophysiological work on the visual and auditory systems of mammals to specific perceptual functions, it is necessary to interrelate electrophysiological and psychophysical data. The great majority of psychophysical experiments in vision and audition that figure in this effort are like our experiments in that they study trade-offs between various parameters of the stimuli. The present section explicates why trade-off experiments have played and will continue to play a central role in efforts to construct neurobehavioral linkage hypotheses-hypotheses of the form: "This anatomically and electrophysiologically defined neural system carries the rewarding signal in self-stimulation."

\section{How Behavioral Trade-Off Experiments Place Constraints on the Underlying System}

A trade-off experiment establishes combinations of two stimulus parameters that produce equivalent behavioral responses. For example, the spectral sensitivity experiment establishes combinations of wavelength and intensity that produce the same frequency of Yes, I see it responses. There are very many neurophysiologically distinct links in the chain of cause and effect that translates the light stimulus into a verbal response. The first is the isomerization of 
rhodopsin; the second is the effect of isomerized rhodopsin on some as yet unknown intrarod "transmitter substance"; the third is the action of this transmitter substance on the ionic gates in the rod membrane; and so on, through the retina to the brain and ultimately to the speech musculature. However, given one condition-monotonicitythe trade-off function determined by observing the verbal response must also apply when one observes instead the output at any one of the links in the neurophysiological chain from light input to verbal output. The behavioral trade-off function propagates backwards, so to speak. It applies at every stage in the underlying succession of causes and effects.

The function relating behavioral output to stimulus input is a composite of the functions describing each successive stage. To illustrate, let $f_{\mathrm{b}}(I, \lambda)$ be the function relating the frequency of Yes I see it responses to the intensity $I$ of a light at wavelength $\lambda$; let $f_{1}$ $(I, \lambda)$ be the function relating the amount of isomerized rhodopsin $R$ to the intensity of light at a given wavelength; let $f_{2}(R)$ be the function relating the amount of intrarod transmitter substance $T$ to the amount of isomerized rhodopsin; and so on, for $f_{3}, f_{4}$, $\ldots f_{n}$. Each successive $f$ is the function that describes the next stage in the genesis of a perception of light. Then

$$
f_{\mathrm{b}}(I, \lambda)=f_{n} \circ f_{n-1} \circ \ldots \circ f_{2} \circ f_{1}(I, \lambda),
$$

where - denotes composition of functions, that is, the operation of letting the ouput of function $i$ be the input to function $i+1$.

The function $f_{b}(I, \lambda)$ is monotonic with respect to $I$ only if each of the functions $f_{I}$ through $f_{n}$ is one to one. (If one stipulates that only continuous functions may be considered, then $f_{\mathrm{b}}$ is monotonic if and only if each of its constituents is also monotonic.) To each output from a one to one function, there corresponds one and only one input. Thus, to each frequency of seeing, there corresponds one and only one value of $f_{n}$, one and only one value of $f_{n-1}$, and so on back to $f_{1}$. Therefore, each combination $\left(I_{i}, \lambda_{i}\right)$ that produces a given frequency of seeing must isomerize the same amount of rhodopsin as any other combination $\left(I_{j}, \lambda_{j}\right)$ that produces the same frequency of seeing. When one determines the combinations of wavelength and intensity that produce the same frequency of seeing, one is determining the combinations that produce the same amount of isomerized rhodopsin. One is also determining the combinations that produce the same amount of intrarod transmitter substance, the same effect on the rod membrane, and so on for every stage. The behavioral trade-off function must be manifest in the behavior-relevant output of every stage in the system.

This reasoning explains the remarkable fact that the trade-off function derived from human verbal reports reflects what is, in essence, a quantum mechanical property of the rhodopsin molecule. The human verbal report is a remote consequence of the isomerization of rhodopsin. Many, many other processes intervene before this isomerization eventuates in a verbal report. We know nothing about most of the intervening processes. Nonetheless, the in situ action spectrum of the rhodopsin molecule is superimposable on the scotopic spectral sensitivity curve of the human observer.

Most important from the perspective of this review, if the in situ action spectrum of rhodopsin were not the same as the scotopic spectral sensitivity curve, one would have to conclude that the isomerization of rhodopsin was not the first stage in scotopic vision or at least not the entire first stage. Congruence with the scotopic spectral sensitivity curve is a constraint that the vision-relevant output of the first stage must satisfy. Any hypothesis about the first stage of light transduction that does not satisfy this constraint must be rejected. The function of behavioral trade-off data in the reductionist enterprise is to establish characteristics that neurophysiological processes must satisfy. In the absence of such data, it is difficult to bring neurophysiological data to bear in any very telling way on the question of what constitutes the neural substrate for a behavioral phenomenon.

\section{Univariance Versus Multivariance}

There is an implicit assumption in the above argument, namely, that the initial physiological process maps the two stimulus 
parameters into a single physiological variable. In vision, this assumption is equivalent to the assumption that there is only one photopigment mediating scotopic vision, which is the univariance hypothesis. If, as in photopic vision (bright-light or color vision), the first stage maps the input into two or more independent physiological variables, then the situation is more complicated. The behavioral trade-off function is no longer a constraint on any single physiological variable. Rather, it is a constraint on the population of independent physiological variables that operate in parallel at any one stage in the system.

Two physiological variables (e.g., two neural signals) are independent if there cannot be in principle a rule for deriving the value (e.g., strength) of one variable given the value of the other. Conversely, two physiological variables are reciprocally dependent if there exists in fact or must exist in principle a rule for deriving the value of one given the value of the other and the information that both variables are being driven by the same stimulus.

The signals from homogenously illuminated rods and from all subsequent neurons driven by homogenously illuminated rods are reciprocally dependent. They are all functions, albeit distinct functions, of the same variable, namely rhodopsin. Therefore, there must exist in principle a function that specifies the strength of the signal from one rod given the strength of the signal from any other rod illuminated by the same light. Combinations, $(I, \lambda)$, that are equivalent for one rod must be equivalent for any other rod.

On the other hand, the signals from two homogenously illuminated cones with differing action spectra are independent. There are combinations of wavelength and intensity that produce the same signal from one cone but different signals from the other. Therefore, there cannot exist a function for determining the signal from one cone given only the signal from the other cone (and the information that both cones are illuminated by the same light). One must know the actual wavelength and intensity of the incident light.

In sum, signals from cones with distinct action spectra are independent physiological variables; they do not covary when both the intensity and wavelength of the light are varied. Signals from rods are reciprocally dependent physiological variables. Since the driving process-the isomerization of rhodopsin-has the same action spectrum from one rod to the next, rod signals necessarily covary when wavelength and intensity are varied.

An important corollary of the definition of independent physiological variables is that if any stage in a neurobehavioral system is a univariate stage, then all subsequent stages are also univariate. That is, if all the neural signals at one stage are reciprocally dependent signals, then the neural signals that mediate any subsequent stage must also be reciprocally dependent (i.e., inferrable one from another). If all the neural signals mediating a given stage are reciprocally dependent, then that stage is a univariate stage, and vice versa.

In a univariate system, the behavioral trade-off function may be directly compared to the trade-off function for each physiological variable. Univariate stages have the convenient property that the trade-off function for the stage as a whole must also be the trade-off function for each individual physiological variable. The part must behave just like the whole.

Given a multivariate stage, the behaviorally derived trade-off function does not constrain any one of the independent variables. When the trade-off function for any one variable fails to match the behavioral tradeoff function, the failure is not grounds for rejecting the hypothesis that the variable is part of a mediating stage. The fact that the action spectrum of the short-wavelength cones does not match the photopic action spectrum is not a reason for assuming that these cones play no mediating role in photopic vision. For a multivariate stage, the constraint is that the behavioral trade-off function must be a combination of the tradeoff functions determined separately for each independent physiological variable. For example, the color-matching functions must be some combination of the three distinct action spectra from the three cone types (Pugh \& Sigel, 1978).

The existence of several independent phys- 
iological variables operating in parallel greatly complicates the assessment of whether or not the events at some neural stage satisfy the quantitative constraints imposed by the behavioral data. In a multivariate system, the behavioral trade-off function may only be compared to the function generated by combining in some way the trade-off functions for each independent physiological variable. Such comparisons are a test of two distinct hypotheses, both of which must be correct in order for the physiologically defined function to agree with the behaviorally defined function. The first hypothesis is that one has found the right physiological variables, the variables that are part of the causal chain in a neurobehavioral system. The second hypothesis is that one has specified the right combination of those variables. It is the confounding of these two hypotheses in dealing with multivariate systems that makes it more difficult to assess whether or not the physiologically defined trade-off data are congruent with the behavioral trade-off function. This may be illustrated by considering the problem of relating Matthews's (1978) electrophysiological data to his behavioral strengthduration functions.

Suppose that Matthews's electrophysiological data encouraged the assumption that he was recording from neurons in the population that form the reward pathway, how would one make a more rigorous assessment of the extent to which the single-unit functions were consistent with the behavioral strength-duration function? The simplest way for the electrophysiological data to be consistent with the behavioral data would be for each axon in the pathway to have the same strength-duration function as was obtained in the behavioral experiments. That is, when one determined the trade-off between the duration of a stimulating pulse from the rewarding electrode and the current required for that pulse to fire an action potential, one found that the resulting functions were superimposable on the behavioral function. To evaluate the single unit data in this straightforward way is to assume that the cable is a univariate stage. One assumes, in other words, that each axon in the cable has the same strength-duration function, in which case the behavioral function directly reflects the single unit functions.

It is, however, entirely possible that the cable is not a univariate stage: The behavioral function may reflect some composite of characteristics that differ from axon to axon within the cable. To evaluate single unit data in the light of this possibility, one must make assumptions about how the next stage of the system weights action potentials across axons and about how the next stage weights successive action potentials within the same axon. Data discussed above justify the assumption that with the duration of a train of stimulating pulses fixed, all the next stage responds to is the number of action potentials in the barrage delivered by the cable. Given this assumption, one can now compute from the single unit data a population trade-off function. This function will specify for each pulse duration the current required to produce a given number of action potentials in the population of axons one has recorded from. Axons that have short-chronaxie functions will contribute disproportionately to the population function at short pulse durations. That is, firing of these axons will account for a relatively large proportion of the total firings at short durations. Conversely, axons with long-chronaxie functions will contribute disproportionately to the population function at long pulse durations.

Another complexity that one may have to deal with is multiple firings. Some or all of the axons one has recorded from may fire more than once when stimulated with long and intense pulses. Each successive pulse in one axon must be treated as a separate physiological variable. That is, one must determine a strength-duration function for eliciting a single firing from that axon, another function for eliciting a second firing, yet another one for eliciting a third firing, and so on. Since, by assumption, the next stage assigns the same weight to an action potential regardless of whether it is the first, second, or $n$th action potential from the axon, the strength-duration functions for secondary and all following firings may be treated just as though they came from another axon in computing the population function.

Conclusion. If there is a large heterogenous population of physiologically defined 
trade-off functions and a great many different ways of combining subsets of them so as to generate functions congruent with the behavioral functions, then the force of the constraint imposed by the behavioral data is negligible. If, on the other hand, the population of physiologically defined trade-off functions is in some way restricted so that only one or a very few combinations of the available functions are congruent with the behavioral data, then the constraints imposed by the behavioral functions have a doubled force: They delimit the set of physiological variables that comprise an intervening stage, and they constrain how those variables are combined by subsequent stages-that is, which variables count for how much under what conditions.

The upshot of this consideration of the multivariate case is that one cannot know in advance how effectively a given behaviorally derived trade-off function will prune the list of possible hypotheses linking physiologically observed variables to a stage in a neurobehavioral system. The pruning force of the constraint depends on the trade-off characteristics of the population of candidate physiological variables. If these characteristics are heterogenous in such a way that there are many ways of combining distinct subsets of the candidates so as to satisfy the behaviorally imposed constraint, then the pruning capacity of the constraint is negligible. One has, in such cases, an embarras de richesse.

Such preliminary electrophysiological data as are available on neural populations that might be thought to mediate self-stimulation suggest that an excess of viable candidates will not be a problem. On the contrary, the problem will be finding a neural system that is in any way capable of satisfying the constraints imposed by the behaviorally derived trade-off data.

\section{The Behavior-Relevant Aspect of a Physiological Variable}

In using trade-off functions to determine whether some physiological variable mediates a particular behavior, one must bear in mind the need to identify the behaviorrelevant aspect of the variable. Take, for example, Bloch's law, which represents the trade-off between the duration and the intensity of a light at "threshold" (i.e., at some arbitrarily chosen frequency of seeing). Assume that the electrical response of the rod membrane is a mediating stage in the perception of dim light flashes. If we now wish to ask whether this electrical response satisfies Bloch's law, we must first guess (or somehow infer) the response-relevant aspect of this electrical response.

It seems likely that very short flashes do not produce electrical responses that are in every way identical to the responses produced by very long flashes (Zacks, 1970). The electrical responses produced by threshold flashes of different duration may be the same only with regard to that aspect that determines whether or not the observer says he sees them. One's first guess might be that the observer's response depended only on the peak of the electrical response. Following up on this guess, one would determine those combinations of flash duration and flash intensity that generated the same peak response. If this trade-off function did not match the behavioral one, one could conclude that the behavioral response was not based on the peak of the electrical response. One could not conclude that the behavioral response did not depend on the electrical response, only that it did not depend on that particular aspect of the electrical response.

Later stages of the visual system might compute a running average of the variance in the rod's electrical potential. The behavioral response might occur when this running average reached some critical level. To be more precise, the behavioral response might depend on the output of a stage that convolved the square of the rod's membrane polarization with a temporal weighting function. In this case, the use of trade-off data would be stymied. One could not perform the appropriate electrophysiological tradeoff experiment unless, of course, one had some way of estimating the temporal weighting function.

The discussion of multivariate stages and response-relevant aspects should make it clear that trade-off functions do not always provide a royal road to the establishment of neurobehavioral linkage hypotheses. None- 
theless, the road they provide, however rocky it may get, will often be among the very few roads there are.

\section{Generality of the Approach}

The experiments we have described constitute a systematic approach to a reductionist problem. Although the mission of physiological psychology is to establish reductionist analyses of behavioral phenomena, the central conceptual problems inherent in such an analysis are seldom explicitly discussed. We take the central question to be how one can justify the claim that a particular set of physiologically and anatomically defined entities is the basis of a behaviorally defined phenomenon. We argue that one answer to this question is to derive from appropriately designed behavioral experiments a set of properties that the physiological and anatomical entities underlying a behavior must possess. Provided this set of properties is a lengthy one that a randomly chosen physiological/anatomical system is unlikely to possess in toto, the demonstration that some coherent physiological/anatomical system does manifest the entire set of properties is evidence for the claim that the system subserves the behavior.

The problem then becomes what constitutes "appropriately designed" behavioral experiments? We argue that trade-off experiments constitute a uniquely powerful class of experiments, which may be applied provided that (a) a behavioral phenomenon is determined by a stimulus that has two or more parameters that may be traded against one another, and (b) there is a monotonic relation between the behavioral output and at least one of the parameters. Trade-off experiments acquire their power from the fact that the trade-off function derived from the behavioral experiment must propagate backward through the underlying sequence of physiological stages. Thus behaviorally determined trade-off functions place quantitative constraints on, and reveal quantitative properties of, the underlying physiological mechanisms.

\section{Significance of Finding Substrate}

What issues in the neurobiology of behavior can be addressed once one has iden- tified the substrate for self-stimulation? Any observer of self-stimulation of the MFB will note three striking aspects of the behavior. The first is the extreme vigor with which the rat strives to obtain more stimulation: It persists hour after hour with great intensity and little apparent fatigue. The second is the extent to which the pursuit of the stimulation excludes the pursuit of other goals. The animal neither eats, nor drinks, nor sleeps for hours on end when allowed continuous access to a self-stimulation lever. The third aspect is most striking when one tests the rat on discrete trials with an appreciable intertrial interval (Gallistel et al., 1974): The animal clearly has a record of the magnitude of the stimulation received on the last several trials. If it has received strongly rewarding stimulation, its performance on a new trial reflects this. If it has received weak or no stimulation, its performance is correspondingly attenuated. The stimulation leaves its mark on the nervous system.

Consider first the vigor of self-stimulation behavior. A rat rendered inactive by a partially paralyzing dose of curare or a subanaesthetic dose of barbiturates will be galvanized by rewarding stimulation (Gallistel et al., Note 4). It will drag itself to the lever and begin to press for more stimulation. As the invigorating effects of successive stimulations summate, the ataxia wanes and the rat begins to resemble an undrugged animal. When the stimulation is turned off, the rat lapses back into a torpor.

Consider next the directing effect of the stimulation. Given a choice between water on one side of the $T$ maze and brain-stimulation reward on the other, the thirsty rat prefers water, unless it has just been stimulated, in which case it chooses more brain stimulation (Deutsch, Adams, \& Metzner, 1964). If the rat is made to wait a minute after being stimulated, it no longer prefers stimulation to water. In short, rewarding stimulation generates a transient but powerful motivational state that selectively potentiates the performance of the stimulationproducing behavior. The transient energizing and directing effect of rewarding stimulation is ordinarily called its priming effect.

Consider last the inclusion in an engram of a record of the magnitude of the brain 
stimulation reward. This record must exist, because the rat's performance on subsequent trials reflects the reward received on earlier trials regardless of the length of the intervening interval. In our minimal model of the substrate for brain stimulation reward, the output of the integrator is a signal that codes the magnitude of reward. Since subsequent performance reflects this magnitude, a neurobiological process must record the output of the integrator for future retrieval.

In sum, self-stimulation manifests in a striking manner three behavioral processes of broad significance: the energizing and directing of behavior and the recording of its consequences. Identifying the neural substrate for self-stimulation should pave the way for further inquiries into the neurobiological bases of these processes.

\section{Reference Notes}

1. Jordan, C. J., Bielajew, C., \& Shizgal, P. Excitability characteristics of the substrate for central grey self-stimulation. Paper presented at meeting of the American Psychological Association, Montreal, Canada, August 1980.

2. Hawkins, R., Roll, P. L., Puerto, A., \& Yeomans, $\mathrm{J}$. The post-stimulation excitability cycle in selfstimulation: A functional measurement analysis. Manuscript in preparation, 1981.

3. Shizgal, P., Howlett, F., \& Corbett, D. Current-distance relationships in rewarding stimulation of the medial forebrain bundle. Paper presented at the meetings of the Canadian Psychological Association, Quebec City, Quebec, 1979.

4. Gallistel, C. R., Boytim, M., Gomita, Y., \& Klebanoff, L. Does pimozide block the reinforcing effect of brain stimulation? The problem of functional specificity in behavioral pharmacology. Manuscript submitted for publication, 1980.

5. Wasserman, E., \& Gallistel, C. R. Pimozide blocks the reinforcing effect but not the priming effect of rewarding brain stimulation. Manuscript submitted for publication, 1980.

6. Kiss, I., Shizgal, P., \& Rosen, H. Electrophysiological characterization of the neural substrate for brain stimulation reward. Unpublished manuscript to be presented at the 1981 meeting of the Canadian Psychological Association.

\section{References}

Adrian, E. D. On the conduction of subnormal disturbances in normal nerve. Journal of Physiology, 1912 , $45,389-412$.

Adrian, E. D. The all-or-none principle in nerve. Journal of Physiology, 1914, 47, 460-474.

Adrian, E. D. The recovery of conductivity and of ex- citability in nerve. Journal of Physiology, 1916, 50, 345-363.

Adrian, E. D., \& Lucas, K. On the summation of a propagated disturbance in nerve and muscle. Journal of Physiology, 1912, 44, 68-124.

Bielajew, C., Jordan, C., Ferme-Enright, J., \& Shizgal, P. Excitability characteristics and anatomical linkage of the substrates for lateral hypothalamic and periaqueductal grey self-stimulation. Physiology and Behavior, in press.

Bielajew, C., \& Shizgal, P. Dissociation of the substrates for medial forebrain bundle self-stimulation and stimulation-escape using a two-electrode stimulation technique. Physiology and Behavior, 1980, 25, 707-712.

Corbett, D., \& Wise, R. A. Intracranial self-stimulation in relation to the ascending dopaminergic systems of the midbrain: A moveable electrode mapping study. Brain Research, 1980, 185, 1-15.

Dennis, S. G., Yeomans, J. S., \& Deutsch, J. A. Adaptation of aversive brain stimulation: III. Excitability characteristics of behaviorally relevant neural substrates. Behavioral Biology, 1976, 18, 531-544.

Deutsch, J. A. Behavioral measurement of the neural refractory period and its application to intracranial self-stimulation. Journal of Comparative and Physiological Psychology, 1964, 58, 1-9.

Deutsch, J. A., Adams, D. W., \& Metzner, R. J. Choice of intracranial stimulation as a function of delay between stimulations and strength of competing drive. Journal of Comparative and Physiological Psychology, 1964, 57, 241-243.

Deutsch, J. A., \& Deutsch, D. Physiological Psychology. Chicago: Dorsey, 1966.

Deutsch, J. A., Roll, P. L., \& Wetter, F. Choice between rewarding brain stimuli of differing length. Behavioral Biology, 1976, 18, 369-377.

Edmonds, D. E., \& Gallistel, C. R. Parametric analysis of brain stimulation reward in the rat: III. Effect of performance variables on the reward summation function. Journal of Comparative and Physiological Psychology, 1974, 87, 876-884.

Edmonds, D. E., \& Gallistel, C. R. Reward versus performance in self-stimulation: Electrode-specific affects of $\alpha$-methyl-p-tyrosine on reward in the rat. Journal of Comparative and Physiological Psychology, 1977, 91, 962-974.

Edmonds, D. E., Stellar, J. R., \& Gallistel, C. R. Parametric analysis of brain stimulation reward in the rat: II. Temporal summation in the reward system. Journal of Comparative and Physiological Psychology, 1974, 87, 860-870.

Ettenberg, A., Cinsavich, S. A., \& White, N. Performance effects with repeated-response measures during pimozide-produced dopamine receptor blockade. Pharmacology, Biochemistry, and Behavior, 1979, $11,557-561$.

Faiers, A. A., \& Mogenson, G. J. Electrophysiological identification of neurons in locus coeruleus. Experimental Neurology, 1976, 53, 254-266.

Feltz, T., \& Albe-Fessard, D. A study of an ascending nigro-caudate pathway. Electroencephalography and Clinical Neurophysiology, 1972, 33, 179-193.

Fibiger, H. C., Carter, D. A., \& Phillips, A. G. Decreased intracranial self-stimulation after neurolep- 
tics or 6-hydroxydopamine: Evidence for mediation by motor deficits rather than by reduced reward. Psychopharmacology, 1976, 47, 21-27.

Fouriezos, G., Hansson, P., \& Wise, R. A. Neuroleptic induced attentuation of brain stimulation reward in rats. Journal of Comparative and Physiological Psychology, 1978, 92, 661-671.

Fouriezos, G., \& Wise, R. A. Pimozide-induced extinction of intracranial self-stimulation. Response patterns rule out motor or performance deficits. Brain Research, 1976, 103, 377-380.

Franklin, K. B. J. Catecholamines and self-stimulation: Reward and performance effects dissociated. Pharmacology, Biochemistry, and Behavior, 1978, 9, 813820.

Franklin, K. B. J., \& McCoy, S. N. Pimozide-induced extinction in rats: Stimulus control of responding rules out motor deficit. Pharmacology, Biochemistry, and Behavior, 1979, 11, 71-75.

Gallistel, C. R. The incentive brain stimulation reward. Journal of Comparative and Physiological Psychology, 1969, 69, 713-721.

Gallistel, C. R. Self-stimulation: The neurophysiology of reward and motivation. In J. A. Deutsch (Ed.), The physiological basis of memory. New York: Academic Press, 1973.

Gallistel, C. R. Motivation as central organizing process: The psychophysical approach to its functional and neurophysiological analysis. In J. Cole \& T. Sonderegger (Eds.), The Nebraska Symposium on Motivation, 1975, 22, 183-250.

Gallistel, C. R. Spatial and temporal summation in the neural circuit subserving brain-stimulation reward. In A. Wauguier \& E. T. Rolls (Eds.), Brain-stimulation reward, New York: Elsevier, 1976.

Gallistel, C. R. Self-stimulation in the rat: Quantitative characteristics of the reward pathway. Journal of Comparative \& Physiological Psychology, 1978, 92, 977-998.

Gallistel, C. R. The organization of action: A new synthesis. Hillsdale, New Jersey: Erlbaum, 1980.

Gallistel, C. R., Rolls, E. T., \& Greene, D. Neuron function inferred by behavioral and electrophysiological measurement of refractory period. Science, 1969 , l66, 1028-1030.

Gallistel, C. R., Stellar, J. R., \& Bubis, E. Parametric analysis of brain stimulation reward in the rat: $I$. The transient process and the memory-containing process. Journal of Comparative and Physiological Psychology, 1974, 87, 848-860.

German, D. C., Dalsass, M., \& Kiser, R. S. Electrophysiological examination of the ventral tegmental (A 10) area in the rat. Brain Research, 1980, 181, 191-197.

Goochee, C., Rasband, W., \& Sokoloff, L. Computerized densitometry and color coding of $\left[{ }^{14} \mathrm{C}\right]$ deoxyglucose autoradiographs. Annals of Neurology, 1980, 7, 359-369.

Guyenet, P. G., \& Aghajanian, G. K. Antidromic identification of dopaminergic and other output neurons of the rat substantia nigra. Brain Research, 1978 , $150,69-84$.

Hill, A. V. Excitation and accommodation in nerve.
Proceedings of the Royal Society of London, Series B. 1936, 119, 305-355.

Hodgkin, A. L., \& Huxley, A. F. The dual effect of membrane potential on sodium conductance in the giant axon of Loligia. Journal of Physiology, 1952. 116, 497-506.

Hoorweg, L. Über die elektrische Nervenerregung. Pflugers Archiv für die gesamte Physiologie, 1892, 52, 87-108.

Hursch, J. B. Conduction velocity and diameter of nerve fibers. American Journal of Physiology, 1939, 127, 131-139.

Kocsis, J. D., Swadlow, H. A., Waxman, S. G., \& Brill, M. H. Variation in conduction velocity during the relative refractory and supernormal periods: $A$ mechanism for impulse entrainment in central axons. $E x$ perimental Neurology, 1979, 65, 230-236.

König, J. F. R., \& Klippel, R. A. The rat brain: $A$ sterotaxic atlas. Baltimore: Williams \& Wilkins, 1967. (Facsimile edition now published by R. E. Krieger, Huntington, N.Y.)

Lapicque, L. Recherches quantitatives sur l'excitation électrique des nerfs traitée comme une polarisation. Journal de Physiologie et de Pathologie Générale, $1907,9,620-635$.

Lucas, K. The effect of alcohol on the excitation, conduction, \& recovery process in nerve. Journal of Physiology, 1913, 46, 470-505.

Matthews, G. Neural substrate for brain stimulation reward in the rat: Cathodal and anodal strength-duration properties. Journal of Comparative and Physiological Psychology, 1977, 91, 858-874.

Matthews, G. Strength-duration properties of single units driven by electrical stimulation of the lateral hypothalamus in rats. Brain Research Bulletin, 1978, 3, 171-174.

Miliaresis, E., \& Rompré, P-P. Self-stimulation and circling: Differentiation of the neural substrata by behavioral measurement with the use of the doublepulse technique. Physiology and Behavior, 1980, 25 939-944.

Milner, P. M. Test of two hypotheses about summation of rewarding brain stimulation. Canadian Journal of Psychology, 1978, 32, 95-105.

Nauta, W. J. H., \& Haymaker, W. Hypothalamic nuclei and fiber connections. In W. Haymaker, E. Anderson, \& W. J. H. Nauta (Eds.), The hypothalamus. Springfield, Ill.: Charles C Thomas, 1969.

Norman, M. F., \& Gallistel, C. R. What can one learn from a strength-duration experiment? Journal of Mathematical Psychology, 1978, 18, 1-24.

Olds, J. A. A preliminary mapping of electrical reinforcing effects in the rat brain. Journal of Comparative and Physiological Psychology, 1956, 49, 281 285.

Phillips, A. G., \& Fibiger, H. C. Decreased resistance to extinction after haloperidol: Implications for the role of dopamine in reinforcement. Pharmacology, Biochemistry, and Behavior, 1979, 10, 751-760.

Pugh, E. N., \& Sigel, C. Evaluation of the candidacy of the I-Mechanisms of Stiles for color-matching fundamentals. Vision Research, 1978, 18, 317-330.

Ranck, J. B., Jr. Which elements are excited in electrical 
stimulation of mammalian central nervous system: A review. Brain Research, 1975, 98, 417-440.

Rolls, E. T. Involvement of brainstem units in medial forebrain bundle self-stimulation. Physiology and Behavior, 1971, 7, 297-310.

Rolls, E. T. The neural basis of brain-stimulation reward. Progress in Neurobiology, 1975, 3, 71-160.

Rolls, E. T., Kelly, P. H., \& Shaw, S. G, Noradrenaline, dopamine, and brain-stimulation reward. Pharmacology, Biochemistry and Behavior, 1974, 2, 735740.

Rushton, W. A. H. Excitable substances in the nervemuscle complex. Journal of Physiology, 1930, 70, 317-337.

Schenk, S., Shizgal, P., \& Bielajew, C. A comparison between the excitability characteristics of the substrates for lateral hypothalamic and frontal cortex self-stimulation. Society for Neuroscience Abstracts, $.1980,6,423$ (Nol47.21).

Segal, M., \& Bloom, F. E. The action of norepinephrine on the rat hippocampus. II. Activation of the input pathway. Brain Research, 1974, 72, 99-114.

Shizgal, P. Electrical brain stimulation in the rat. Differentiation of temporal integration characteristics in the substrates for the rewarding and aversive effects. Doctoral dissertation, University of Pennsylvania, 1975

Shizgal, P., Bielajew, C., Corbett, D., Skelton, R., \& Yeomans, J. Behavioral methods for inferring anatomical linkage between rewarding brain stimulation sites. Journal of Comparative and Physiological Psychology, 1980, 94, 227-237.

Shizgal, P., Bielajew, C., \& Yeomans, J. S. Behaviorally derived estimates of conduction velocity and refractory period in a reward-related pathway differ from the characteristics of monaminergic neurons. Society for Neuroscience Abstracts, 1979, 5, 352 (No. 1176).

Shizgal, P., \& Matthews, G. Electrical stimulation of the rat diencephalon: Differential effects of interrupted stimulation on on- and off-responding. Brain Research, 1977, 129, 319-333.

Skelton, R. W., \& Shizgal, P. Parametric analysis of on- and off-responding for hypothalamic stimulation. Physiology and Behavior, 1980, 25, 699-706.

Sokoloff, L. et al. The ${ }^{14} \mathrm{C}$-deoxyglucose method for measurement of local cerebral glucose utilization:
Theory, procedure, and normal values in the conscious and anesthetized albino rat. Journal of Neurochemistry, 1977, 28, 897-916.

Swadlow, H. A., \& Waxman, S. G. Variations in conduction velocity and excitability following single and multiple impulses of visual callosal axons in the rabbit. Experimental Neurology, 1976, 53, 128-150.

Szabo, I., Lénard, L., \& Kosaras, B., Drive decay theory of self-stimulation: Refractory periods and axon diameters in hypothalamic reward loci. Physiology and Behavior, 1974, 12, 329-343.

Takigawa, M., \& Mogenson, G. J. A study of inputs to antidromically identified neurons of the locus coeruleus. Brain Research, 1977, 135, 217-230.

Waxman, S. G., \& Bennett, M. V. L. Relative conduction velocities of small myelinated fibers in the central nervous system. Nature, 1972, 238, 217-219.

Waxman, S. G., \& Swadlow, H. A. Ultrastructure of visual callosal axons in the rabbit. Experimental Neurology, 1976, 53, 115-127.

Wise, R. A. Catecholamine theories of reward: A critical review. Brain Research, 1978, 152, 215-247.

Yeomans, J. S. Quantitative measurement of neural post-stimulation excitability with behavioral methods. Physiology and Behavior, 1975, 15, 593-602.

Yeomans, J, S. The absolute refractory periods of selfstimulation neurons. Physiology and Behavior, 1979, 22, 911-919.

Yeomans, J.S., \& Davis, J. K. Behavioral measurement of the post-stimulation excitability of neurons mediating self-stimulation by varing the voltage of paired pulses. Behavioral Biology, 1975, 15, 435-447.

Yeomans, J. S., Matthews, G. G., Hawkins, R. D., Bellman, K., \& Doppelt, H. Characterization of self-stimulation neurons by their local potential summation properties. Physiology and Behavior, 1979, 22, 921 929.

Yim, C. Y., \& Mogenson, G. J. Electrophysiological studies of neurons in the ventral tegmental area of Tsai. Brain Research, 1980, 181, 301-313.

Zacks, J. L. Temporal summation phenomena at threshold: Their relation to visual mechanisms. Science, $1970,170,197-199$.

Received March 18, 1980 Revision received August 8, 1980 